\title{
Electric Field Autocorrelation Functions for Beginning Multiple Rayleigh Scattering
}

James A. Lock

Cleveland State University, j.lock@csuohio.edu

Follow this and additional works at: https://engagedscholarship.csuohio.edu/sciphysics_facpub

Part of the Physics Commons

How does access to this work benefit you? Let us know!

\section{Publisher's Statement}

This paper was published in Applied Optics and is made available as an electronic reprint with the permission of OSA. The paper can be found at the following URL on the OSA website: http://www.opticsinfobase.org/ao/abstract.cfm?URI=ao-40-24-4187. Systematic or multiple reproduction or distribution to multiple locations via electronic or other means is prohibited and is subject to penalties under law.

\section{Original Citation}

Lock, James A. "Electric Field Autocorrelation Functions for Beginning Multiple Rayleigh Scattering." Applied Optics 40 (2001): 4187-4203.

\section{Repository Citation}

Lock, James A., "Electric Field Autocorrelation Functions for Beginning Multiple Rayleigh Scattering" (2001). Physics Faculty Publications. 19.

https://engagedscholarship.csuohio.edu/sciphysics_facpub/19

This Article is brought to you for free and open access by the Physics Department at EngagedScholarship@CSU. It has been accepted for inclusion in Physics Faculty Publications by an authorized administrator of EngagedScholarship@CSU. For more information, please contact library.es@csuohio.edu. 


\title{
Electric field autocorrelation functions for beginning multiple Rayleigh scattering
}

\author{
James A. Lock
}

\begin{abstract}
The polarization-resolved electric field autocorrelation function for $p$-order scattering was derived from the order-of-scattering solution of the exact equations for electromagnetic multiple Rayleigh scattering and was calculated for $2 \leq p \leq 6$ for particles undergoing diffusive motion in an idealized sample cell. It was found that the polarization-channel and the scattering-angle dependence of the $p$-order autocorrelation function approximately decoupled from the delay-time dependence for $p \gtrsim 3$. The polarizationchannel and the scattering-angle dependence were analytically calculated, and the delay-time dependence was analytically approximated. The resulting analytical model for the polarization-resolved autocorrelation function for beginning multiple Rayleigh scattering was then tested against experimental autocorrelation data. The data were found to be well fitted by the model. (c) 2001 Optical Society of America

OCIS codes: $\quad 290.0290,290.1990,290.4020,290.4210$.
\end{abstract}

\section{Introduction}

When light is scattered by a collection of small particles that are suspended in a liquid and undergoing Brownian motion the statistics of the particles' random motion are encoded in the temporal fluctuations of the scattered-light intensity. The time scale of the fluctuations is obtained from the experimentally measured intensity autocorrelation function. The time scale as well as the functional dependence of the autocorrelation function on the delay time provide information concerning either the size distribution of the suspended particles or their dynamics.

The autocorrelation function assumes a simple form in both the low- and the high-concentration limits. For low concentrations light is scattered only once by the suspended particles before leaving the sample cell. The autocorrelation function in this case is a decaying exponential in the delay time, with the decay constant proportional to the particle size. ${ }^{1}$ For high concentrations of suspended particles that are not index matched to the surrounding liquid light is scattered tens or hundreds of times by the particles before leaving the sample cell. In this deep

The author (jimandcarol@stratos.net) is with the Department of Physics, Cleveland State University, Cleveland, Ohio 44115.

Received 5 October 2000; revised manuscript received 1 May 2001.

0003-6935/01/244187-17\$15.00/0

(C) 2001 Optical Society of America multiple-scattering limit both the polarizationchannel and the scattering-angle dependence of singly scattered light are thoroughly homogenized by the large number of scatterings. The autocorrelation function for transmission or backscattering by a slab of particles is accurately modeled in the deep multiple-scattering limit by diffusing-wave spectroscopy $^{2}$ (DWS) for which it is assumed that photons from the incident beam diffuse by means of repeated scatterings as they propagate through the sample.

For intermediate concentrations of suspended particles in the regime known as beginning multiple scattering, ${ }^{3}$ or oligo multiple scattering, ${ }^{4}$ light is scattered only a few times before leaving the sample cell, and the autocorrelation function retains some memory of the polarization-channel and the scatteringangle dependence of single scattering. In this regime the electric field autocorrelation function is often modeled by use of the order-of-scattering approach in which individual autocorrelation functions for single scattering, double scattering, triple scattering, etc., are calculated, weighted by the relative strength of each order of multiple scattering, and added together. ${ }^{5-10}$ Double-scattering autocorrelation functions have been calculated for a number of different systems.,3,5-10 Higher-order multiplescattering autocorrelation functions have often been modeled by increasingly rapidly decaying exponentials in the delay time, and the relative strength of various-order scatterings has often been modeled by Poisson statistics. ${ }^{6,7}$ The beginning multiple- 
scattering regime has also been investigated by use of radiative transfer methods, ${ }^{3}$ DWS-type techniques, ${ }^{4,11,12}$ Monte Carlo techniques, ${ }^{13}$ and computer modeling of the particles' Brownian motion along with exact electromagnetic wave multiple-scattering theory. ${ }^{14}$

In this paper a simple analytic approximation to the polarization-resolved electric field autocorrelation function for beginning multiple Rayleigh scattering is derived and compared with experimental data. The principal results are as follows:

(i) The polarization-resolved autocorrelation function is written as the contribution of single scattering plus that of double scattering plus that of triple scattering, and so on. All the order-of-scattering contributions are simple analytical functions and are straightforward to program on a computer.

(ii) The approximate autocorrelation function takes the form of a series in powers of a quantity $Q$ that depends in a simple way on the wavelength of light, the radius, the refractive index, and the volume fraction of the suspended particles, and the size of the sample cell.

(iii) Increased multiple scattering reduces the polarization-channel and the scattering-angle dependence of the autocorrelation function. This dependence is greatest for double scattering and progressively diminishes for triple scattering, quadruple scattering, and so on.

(iv) Experimental data are found to be well fitted by the approximate autocorrelation function.

What is novel about the approach taken here is that the derivation of the approximate autocorrelation function is based on the iteration solution of the exact equations ${ }^{15,16}$ for multiple Rayleigh scattering of electromagnetic waves. The case of multiple Rayleigh scattering is studied here to simplify as much as possible the partial-wave sums and the vector spherical harmonic-translation coefficients appearing in the exact equations for multiple scattering of an electromagnetic wave by a collection of arbitrary-sized particles. Although Rayleigh scattering is the simplest example of electromagnetic scattering, it is still sufficiently complicated that the single-scattering intensity exhibits both polarization-channel and scattering-angle dependence. As a result, multiple Rayleigh scattering serves as both a physically interesting and a mathematically tractable prototype system for the study of multiple scattering of electromagnetic waves by a collection of particles.

The results presented here suggest that an important feature of the multiple-scattering autocorrelation function is separability. In the beginning multiple-scattering regime, the electric field autocorrelation function for each scattering order depends on the incident-scattered polarization channel, the scattering angle, and the delay time. To a good approximation the autocorrelation function for each scattering order separates into a factor that depends solely on the polarization channel and the scattering angle and that is multiplied by a second factor that depends solely on the delay time.

The body of this paper is organized as follows: In Section 2 the iteration solution of the exact equations for electromagnetic multiple Rayleigh scattering is obtained. This is accomplished in a number of steps. In Subsection 2.A the geometry of the incident beam, the sample cell, and the detector is described. In Subsection 2.B the exact equations for multiple scattering of an electromagnetic wave by a collection of arbitrary-sized spheres located at known positions is given in symbolic form. In Subsection 2.C the equations are simplified for the case of polarizationresolved multiple Rayleigh scattering to which only the partial wave $L=1$ contributes. In Subsection 2.D the multiple Rayleigh scattering equations are iterated to obtain the electric field arriving at the detector following $p$ scatterings of electromagnetic waves by the particles. In Subsection 2.E the iteration solution to the exact multiple-scattering equations is derived for $s$-wave (i.e., isotropic) scattering of scalar waves to assess the importance of polarization and single-scattering anisotropy in multiple Rayleigh scattering.

In Sections 3-5 the approximate autocorrelation function for beginning multiple scattering is derived. Again, this is accomplished in a number of steps. First, in Subsections 3.A and 3.B the electric field autocorrelation function for multiple Rayleigh scattering and the amplitude autocorrelation function for multiple $s$-wave scattering of scalar waves, respectively, are ensemble averaged. In each case it is assumed that the particles undergo Brownian motion in a scattering cell whose idealized geometry is chosen to maximize the portion of the ensemble averaging that can be performed analytically rather than to model an experimental geometry faithfully. An advantage of this approach is that the autocorrelation function may be calculated for relatively high numbers of scatterings to identify trends in the results, in particular the decoupling of the delay-time dependence from the polarization-channel and the scattering-angle dependence. In Section 4, the zerodelay-time limit of the polarization-resolved autocorrelation functions is examined, and a simple pair of recursion relations is derived for the polarizationchannel and the scattering-angle dependence of the intensity for each order of multiple scattering. This serves as a normalizing factor for the $p$-order autocorrelation functions.

In Subsections 5.A and 5.B the delay-time dependence of the normalized electric field autocorration functions and the multiple $s$-wave scattering autocorrelation function for scalar waves is examined. As the scattering order increases, it is found that the normalized electric field autocorrelation functions exhibit increasingly less polarization-channel and scattering-angle dependence and become increasingly better approximated by the $s$-wave autocorrelation function, which in turn in Subsection 5.C is analytically approximated. Finally, in Section 6 these results are combined into a simple analytical 


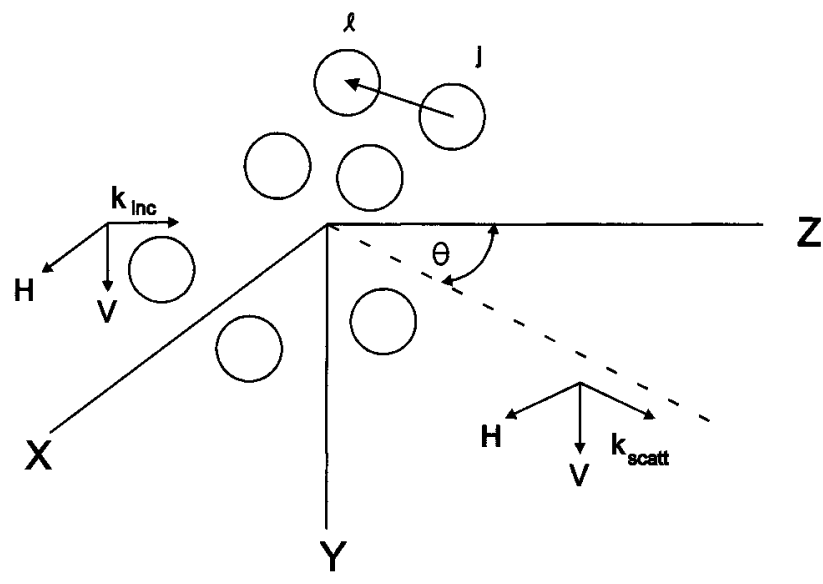

Fig. 1. Plane wave with the wave vector $\mathbf{k}_{\text {inc }}$ incident upon a collection of spherical particles: Light scattered at the angle $\theta$ in the $x z$ plane of the $x, y, z$ laboratory coordinate system has the wave vector $\mathbf{k}_{\text {scatt. }}$. The position of particle $\ell$ with respect to particle $j$ is also shown, as is the $V$ and the $H$ polarization direction of the incoming and the outgoing electric field.

model for the polarization-resolved electric field autocorrelation function for beginning multiple Rayleigh scattering. The model is then tested against experimental autocorrelation data.

\section{Exact Multiple-Scattering Theory}

\section{A. Scattering Geometry}

Consider a linearly polarized electromagnetic plane wave that is multiply scattered by a collection of $J$ spherical particles that are suspended in a liquid of refractive index $m$ in a roughly equidimensional sample cell of characteristic radius $R_{\text {ave }}$. For convenience, the particles are taken to be identical, having a radius $a$ and an arbitrary real refractive index $n$ relative to the liquid. The positions of the particles are $\mathbf{r}_{j}$ for $1 \leq j \leq J$. The volume fraction $f$ of the suspended particles is small enough that the particle positions are independent, and pair-correlation effects and hydrodynamic interactions are not important. 2,5 The plane wave has the time dependence $\exp (-i \omega t)$, electric field strength $E_{0}$, wavelength $\lambda$, and wave number $k=2 \pi m / \lambda$ in the liquid. The wave propagates in the $z$ direction and is polarized either in the $x$ direction or in the $y$ direction, hereafter called $H$ for horizontal and $V$ for vertical, respectively, as is shown in Fig. 1. The detector, whose field of view encompasses the entire sample cell, is located at the angles $\theta, \phi$ in the scattering far zone at a distance $r$ from the origin of coordinates, which is placed at the center of the cell. The distance $r$ is sufficiently large that light scattered only in the $\theta, \phi$ direction by each particle is recorded at the detector.

This geometry differs considerably from the usual geometry of dynamic light-scattering experiments in which a tightly focused laser beam passes through the sample cell and illuminates only a small fraction of the particles within it, and the detector has a narrow field of view that intersects the beam inside the cell. The usual geometry is designed to minimize the effects of multiple scattering because single scattering is strong in the intersection volume and multiple scattering is weak along the detector's narrow field of view. For the geometry considered here, all the particles in the sample cell are illuminated by the plane wave, and the wide-field-of-view detector maximizes the amount of multiply scattered light that is detected.

To simplify the ensemble averaging described in Section 3 greatly, let us assume that each suspended particle in the sample cell is surrounded in all directions by a random distribution of other particles. Further, we assume that the radiation incident upon the suspended particle was emitted by other particles located up to a distance $R_{\text {ave }}$ away from it and was not scattered by any of the intervening particles along the way. We call this very idealized assumption the isotropic-environment assumption. It is approximately satisfied for strong scattering for which $R_{\text {ave }}$ is the single-scattering mean free path and for particles near the center of the sample cell for weak scattering for which $R_{\text {ave }}$ is the cell radius. This assumption does not hold for particles near the surface of the sample cell. But if the cell is sufficiently large that its volume-to-surface-area ratio is also large the isotropic-environment assumption can be considered approximately valid for the entire collection of suspended particles. This reasoning constitutes a purely geometrical argument and is distinct from the dynamic particle-sample cell-wall interaction whose range is typically less than $0.3 \mu \mathrm{m}$.

\section{B. Exact Electromagnetic Multiple-Scattering Equations}

In Mie scattering theory the exact electromagnetic fields scattered by a single spherical particle are written as a partial-wave sum of spherical multipole waves. The amplitude of each scattered multipole is obtained from the fields incident upon the particle by one's matching the boundary conditions for the tangential components of the incident, the scattered, and the interior electric and magnetic fields at the particle's surface. The electric field incident upon particle $j$ and the field scattered by it are related by

$$
\mathbf{E}_{\text {scattered by } j}(\mathbf{r})=M_{j}\left[\mathbf{E}_{\text {incident on } j}\left(\mathbf{r}_{j}\right)\right]
$$

where $\mathbf{r}$ is an arbitrary position and $M_{j}$ is the Mie scattering operator for particle $j$. The partial-wave sum in Eq. (1) was suppressed. If the incident plane wave is scattered by a collection of $J$ particles the total outgoing electric field is

$$
\mathbf{E}^{\text {total }}(\mathbf{r})=\mathbf{E}_{\text {beam }}(\mathbf{r})+\sum_{j=1}^{J} \mathbf{E}_{\text {scattered by } j}(\mathbf{r}),
$$

where $\mathbf{E}_{\text {beam }}$ is the electric field of the plane wave. For single scattering the plane wave alone initiates scattering at particle $j$ in Eq. (1). But for multiple scattering the electric field incident upon particle $j$ 
also includes the scattered fields of all the other particles ${ }^{17,18}$ :

$$
\mathbf{E}_{\text {incident on } j}\left(\mathbf{r}_{j}\right)=\mathbf{E}_{\text {beam }}\left(\mathbf{r}_{j}\right)+\sum_{\substack{\ell=1 \\ \ell \neq j}}^{J} \mathbf{E}_{\text {scattered by } l}\left(\mathbf{r}_{j}\right) .
$$

The expressions relating the incident and the scattered magnetic fields are identical. The solution of Eqs. (1)-(3) along with the corresponding magnetic field equations provide an exact description of the multiple scattering of an electromagnetic plane wave by a collection of particles at known positions.

But actually solving these equations for a specific collection of particles is greatly complicated by the fact that the fields in the partial-wave sums implicit in Eqs. (1)-(3) are expressed in different coordinate systems. The incident and the scattered multipole waves in Eq. (1) are written in terms of the coordinate system with the center of particle $j$ at the origin. The total field in Eq. (2), on the other hand, is written in terms of the laboratory coordinate system of Fig. 1. Thus the multipole waves scattered by particle $j$ in Eq. (1) must first be rewritten in terms of the laboratory coordinates before Eq. (2) can be used. This can be accomplished by use of the vector spherical harmonic-translation coefficients. ${ }^{19,20}$ Likewise, for Eq. (3) to be used as input to Eq. (1) the spherical multipoles of both the incoming plane wave and the multipole waves scattered by particle $\ell$ must first be transformed from the laboratory coordinate system and the coordinate system centered on particle $\ell$, respectively, to the coordinate system centered on particle $j$.

Some of these changes of coordinate system are relatively uncomplicated. When the detector is in the scattering far zone the vector spherical harmonictranslation coefficients relating the laboratory coordinate system and the coordinate system centered on particle $j$ reduce to a simple phase shift, $\exp \left(-i \mathbf{k}_{\text {scatt }}{ }^{\circ}\right.$ $\mathbf{r}_{j}$ ), of each multipole wave, ${ }^{21}$ where the wave vector of the scattered light reaching the detector is

$$
\mathbf{k}_{\mathrm{scatt}}=k\left(\sin \theta \cos \phi \mathbf{u}_{x}+\sin \theta \sin \phi \mathbf{u}_{y}+\cos \theta \mathbf{u}_{z}\right) .
$$

The physical interpretation of this phase is that, because different particles are different distances from the detector, light must be scattered by them at different times to reach the detector simultaneously. The transformation of the spherical multipoles of the incoming plane wave from the laboratory coordinate system to the coordinate system centered on particle $j$ is also a simple phase $\operatorname{shift}, \exp \left(i \mathbf{k}_{\text {inc }} \cdot \mathbf{r}_{j}\right)$, where the wave vector of the incident beam is

$$
\mathbf{k}_{\text {inc }}=k \mathbf{u}_{z} .
$$

Equation (5) is the phase of the incident plane wave at particle $j$ with respect to the phase at the origin of the laboratory coordinates. But because particles $\ell$ and $j$ are relatively close to each other, the vector spherical harmonic-translation coefficients for trans- forming $\mathbf{E}_{\text {scattered by } \ell}$ from the $\ell$ coordinate system to the $j$ coordinate system cannot, in general, be simplified. The exact electromagnetic scattering equations containing all the sums over partial waves and vector spherical harmonic-translation coefficients are given in Refs. 15, 16, and 21.

\section{Multiple Rayleigh Scattering}

The exact multiple-scattering equations simplify considerably in the Rayleigh scattering limit of $k a \ll 1$, where the scattered field of each particle contains only the $L=1$ partial wave corresponding to electric dipole radiation. The Rayleigh scattering amplitude of each particle is ${ }^{22}$

$$
a_{1}=(-2 i / 3)\left[\left(n^{2}-1\right) /\left(n^{2}+2\right)\right](k a)^{3} .
$$

For $L=1$ radiation leaving particle $\ell$ and exciting $L=1$ radiation at particle $j$ all the vector spherical harmonic-translation coefficients in the exact electromagnetic multiple-scattering equations of Ref. 15 can be straightforwardly evaluated. The resulting fields that propagate from particle $\ell$ to particle $j$, given in Eqs. (10) and (11) below, are a mixture of $L=0$ and $L=2$ multipole fields in the reference frame of $j$, in accord with the triangle inequality for the coupling of angular momentum.

In the scattering far zone and in the laboratory coordinate system of Fig. 1 the exact multiple Rayleigh scattering equations are

$$
\begin{aligned}
\mathbf{E}_{\text {scattered by } j}(r, \theta, \phi)= & {[i \exp (i k r) /(k r)] } \\
& \times\left[S_{2}(\theta, \phi) \mathbf{u}_{\theta}-S_{1}(\theta, \phi) \mathbf{u}_{\phi}\right], \\
\mathbf{B}_{\text {scattered by } j}(r, \theta, \phi)= & {[i \exp (i k r) /(c k r)] } \\
& \times\left[S_{1}(\theta, \phi) \mathbf{u}_{\theta}+S_{2}(\theta, \phi) \mathbf{u}_{\phi}\right],
\end{aligned}
$$

where $c$ is the speed of light and the scattering amplitudes $S_{1}$ and $S_{2}$ are

$$
\begin{aligned}
S_{1}(\theta, \phi)= & -P_{+} \cos \phi+P_{-} \sin \phi, \\
S_{2}(\theta, \phi)= & P_{+} \cos \theta \sin \phi-P_{0} \sin \theta \\
& +P_{-} \cos \theta \cos \phi,
\end{aligned}
$$

with

$$
\begin{gathered}
P_{+}=\sum_{j=1}^{J} \exp \left(-i \mathbf{k}_{\mathrm{scatt}} \cdot \mathbf{r}_{j}\right) P_{+}{ }^{j}, \\
P_{0}=\sum_{j=1}^{J} \exp \left(-i \mathbf{k}_{\mathrm{scatt}} \cdot \mathbf{r}_{j}\right) P_{0}{ }^{j}, \\
P_{-}=\sum_{j=1}^{J} \exp \left(-i \mathbf{k}_{\mathrm{scatt}} \cdot \mathbf{r}_{j}\right) P_{-}{ }^{j} .
\end{gathered}
$$


In Eqs. (9) the amplitudes $P_{+}{ }^{j}, P_{0}{ }^{j}$, and $P_{-}{ }^{j}$ for particle $j$ are given by the matrix equation

$$
\begin{aligned}
{\left[\begin{array}{c}
P_{+}{ }^{j} \\
P_{0}{ }^{j} \\
P_{-}{ }^{j}
\end{array}\right]=} & (3 / 2) a_{1} E_{0} \exp \left(i \mathbf{k}_{\mathrm{inc}} \cdot \mathbf{r}_{j}\right)\left[\begin{array}{c}
b_{+} \\
b_{0} \\
b_{-}
\end{array}\right] \\
& -i a_{1} \sum_{\substack{\ell=1 \\
\ell \neq j}}^{J} h_{0}^{(1)}\left(k r_{\ell j}\right)\left[\begin{array}{c}
P_{+}{ }^{\ell} \\
P_{0}^{\ell} \\
P_{-}^{\ell}
\end{array}\right] \\
& +(3 i / 2) a_{1} \sum_{\substack{\ell=1 \\
\ell \neq j}}^{J} h_{2}^{(1)}\left(k r_{\ell j}\right)\left[Q_{\ell j}\right]\left[\begin{array}{c}
P_{+}^{\ell} \\
P_{0}^{\ell} \\
P_{-}^{\ell}
\end{array}\right] .
\end{aligned}
$$

The first term on the right-hand side of Eq. (10) describes single scattering, and the second and the third terms describe multiple scattering. The functions $h_{0}^{(1)}$ and $h_{2}^{(1)}$ are spherical Hankel functions of the first kind and correspond to the $L=0$ and the $L=$ 2 portions of the fields, respectively, propagating from particle $\ell$ to particle $j$. The position of particle $\ell$ with respect to particle $j$ is $\mathbf{r}_{\ell j}$, and the electric quadrupole matrix of the $\ell j$ system $\left[Q_{\ell j}\right]$ is ${ }^{23}$

$$
\begin{aligned}
{\left[Q_{\ell j}\right] } & =\left(1 / r_{\ell j}{ }^{2}\right) \\
\times & {\left[\begin{array}{lcc}
\left(r_{\ell j}{ }^{2} / 3\right)-y_{\ell j}{ }^{2} & -y_{\ell j} z_{\ell j} & -x_{\ell j} y_{\ell j} \\
-y_{\ell j} z_{\ell j} & \left(r_{\ell j}{ }^{2} / 3\right)-z_{\ell j}{ }^{2} & -x_{\ell j} z_{\ell j} \\
-x_{\ell j} y_{\ell j} & -x_{\ell j} z_{\ell j} & \left(r_{\ell j}{ }^{2} / 3\right)-x_{\ell j}{ }^{2}
\end{array}\right], } \\
x_{\ell j} & =r_{\ell j} \sin \theta_{\ell j} \cos \phi_{\ell j}, \\
y_{\ell j} & =r_{\ell j} \sin \theta_{\ell j} \sin \phi_{\ell j}, \\
z_{\ell j} & =r_{\ell j} \cos \theta_{\ell j} .
\end{aligned}
$$

Equation (11) depends on only the relative orientation of particles $\ell$ and $j$ and not their separation. If the electric field of the incident plane wave is polarized in the $x$ direction the plane wave is characterized by

$$
b_{+}=0, \quad b_{0}=0, \quad b_{-}=1,
$$

and if the plane wave is polarized in the $y$ direction it is characterized by

$$
b_{+}=1, \quad b_{0}=0, \quad b_{-}=0 .
$$

The multiple Rayleigh scattering equations of Eqs. (7)-(14) are a streamlined version of the equations of the coupled-dipole model ${ }^{24-26}$ for scattering by an agglomeration of small particles. In the coupleddipole model the magnitude and the direction of the induced electric dipole moment of a given particle in the agglomerate is determined by both the incident beam fields and the fields scattered by all the other dipoles. The resulting dipole moment is then substituted along with a rotation of coordinates into a second equation to give the electric dipole scattered fields. Our equations, on the other hand, give the scattered fields directly. Equations (7)-(14) also agree with the multiple Rayleigh scattering equations of Ref. 27, as corrected in Ref. 28, which were derived from a different set of starting assumptions.

The detector is placed in the horizontal $x z$ plane of Fig. 1, and polarization-resolved scattering is considered in which the polarization state of both the incident and the scattered light is either $V$ or $H$. The polarization-resolved multiply scattered electric field then becomes

$$
\begin{aligned}
& \mathbf{E}_{V V}^{\text {total }}(\mathbf{r})=[i \exp (i k r) /(k r)] P_{+} \mathbf{u}_{\phi}, \\
& \mathbf{E}_{V H}^{\text {total }}(\mathbf{r})=[i \exp (i k r) /(k r)]\left(-P_{0} \sin \theta+P_{-} \cos \theta\right) \mathbf{u}_{\theta}, \\
& \mathbf{E}_{H V}^{\text {total }}(\mathbf{r})=[i \exp (i k r) /(k r)] P_{+} \mathbf{u}_{\phi}, \\
& \mathbf{E}_{H H}^{\text {total }}(\mathbf{r})=[i \exp (i k r) /(k r)]\left(-P_{0} \sin \theta+P_{-} \cos \theta\right) \mathbf{u}_{\theta},
\end{aligned}
$$

where the first subscript $H$ or $V$ denotes the polarization of the incoming plane wave and the second subscript denotes the polarization of the multiply scattered light arriving at the detector. For $\mathbf{E}_{V V}^{\text {total }}$ and $\mathbf{E}_{V H}^{\text {total }}$, the amplitudes $P_{+}, P_{0}$, and $P_{-}$are obtained by use of Eqs. (9)-(12) and (14), whereas for $\mathbf{E}_{H V}^{\text {total }}$ and $\mathbf{E}_{H H}^{\text {total }}$ they are obtained by use of Eqs. (9)(13).

Although Eq. (10) can be used to derive the autocorrelation functions for polarization-resolved multiple Rayleigh scattering, the treatment is simplified considerably if one makes the following assumption: The fields incident upon particle $j$ include the scattered fields of all the other particles $\ell$. If the distance between particles $\ell$ and $j$ is large compared with $\lambda$, i.e., $k r_{\ell j} \gg 1$, the electric field scattered by $\ell$ incident upon $j$ can be approximated by its far-zone radiation component, which is proportional to $1 /\left(k r_{\ell j}\right)$. But if the distance between particles $\ell$ and $j$ is much less than a wavelength so that $k r_{\ell j} \ll 1$, the electric field scattered by $\ell$ incident upon $j$ can be approximated by its oscillating electric dipole component, which is proportional to $1 /\left(k r_{\ell j}\right)^{3}$. By computing the ensemble-averaged intensity at particle $j$ that is due to the $h_{2}^{(1)}\left(k r_{\ell j}\right)$ portion of the field of all the other particles $\ell$ within the distance $R_{\text {ave }}$, we find that the $1 /\left(k r_{\ell j}\right)$ component of the field dominates the intensity when

$$
\left(k R_{\text {ave }}\right)(k a)^{3} \gg 1 .
$$

In this case the total field at $j$ that is produced by the neighboring particles, for which $k r_{\ell j} \ll 1$, is negligible compared with that produced by the more distant particles, for which $k r_{\ell j} \gg 1$. For a typical dynamic light-scattering experiment with $\lambda=0.5145 \mu \mathrm{m}, a=$ $0.05 \mu \mathrm{m}, R_{\text {ave }} \approx 2 \mathrm{~mm}$, and $m=1.333$, we have $\left(k r_{\text {ave }}\right)(k a)^{3} \approx 2 \times 10^{4}$, which easily satisfies inequality (16). With this assumption, one can neglect the 
terms in $h_{2}^{(1)}\left(k r_{\ell j}\right)$ that are proportional to $1 /\left(k r_{\ell j}\right)^{2}$ and $1 /\left(k r_{\ell j}\right)^{3}$, and Eq. (10) simplifies to

$$
\begin{aligned}
{\left[\begin{array}{c}
P_{+}{ }^{j} \\
P_{0}{ }^{j} \\
P_{-}^{j}
\end{array}\right]=} & (3 / 2) a_{1} E_{0} \exp \left(i \mathbf{k}_{\mathrm{inc}} \cdot \mathbf{r}_{j}\right)\left[\begin{array}{c}
b_{+} \\
b_{0} \\
b_{-}
\end{array}\right] \\
& +(3 i / 2) a_{1} \sum_{\substack{\ell=1 \\
\ell \neq j}}^{J}\left[\exp \left(k r_{\ell j}\right) /\left(k r_{\ell j}\right)\right]\left[T_{\ell j}\right]\left[\begin{array}{c}
P_{+}^{\ell} \\
P_{0}^{\ell} \\
P_{-}^{\ell}
\end{array}\right],
\end{aligned}
$$

where

$$
\left[T_{\ell j}\right]=\left(1 / r_{\ell j}{ }^{2}\right)\left[\begin{array}{ccc}
x_{\ell j}{ }^{2}+z_{\ell j}{ }^{2} & -y_{\ell j} z_{\ell j} & -x_{\ell j} y_{\ell j} \\
-y_{\ell j} z_{\ell j} & x_{\ell j}{ }^{2}+y_{\ell j}{ }^{2} & -x_{\ell j} z_{\ell j} \\
-x_{\ell j} y_{\ell j} & -x_{\ell j} z_{\ell j} & y_{\ell j}{ }^{2}+z_{\ell j}
\end{array}\right] .
$$

This simplification [Eq. (17)] cannot be used for scattering by a densely packed agglomerate. For example, if $R_{\text {ave }_{3}} \approx 1 \mu \mathrm{m}$ and $a=0.05 \mu \mathrm{m}$, one obtains $\left(k R_{\text {ave }}\right)(k a)^{3} \approx 8.8$, and the effect of the oscillating dipole field of the neighboring particles is substantial.

If one were interested in Mie corrections to Eq. (17) for slightly larger particles the number of coupled scattering amplitudes appearing in the equations would become quite large. The first Mie correction ${ }^{22}$ to Rayleigh scattering consists of the portion of the partial-wave scattering amplitudes $b_{1}$ and $a_{2}$ that are proportional to $(k a)^{5}$ as well as the portion of $a_{1}$ that is proportional to $(k a)^{5}$. These partial-wave scattering amplitudes correspond to the $L=1,2$ terms of the partial-wave sums. The coupling of $L=1$ for particle $\ell$ to $L=1$ for particle $j$ contains $L=0,2$ vector spherical harmonic-translation coefficients, as before. But the coupling of $L=1$ for particle $\ell$ to $L=$ 2 for particle $j$ contains $L=1,3$ terms, and the coupling of $L=2$ for particle $\ell$ to $L=2$ for particle $j$ contains $L=0,2,4$ terms. In addition, the coupling between the $a_{1} / b_{1}$ and the $a_{2} / b_{1}$ terms is crosspolarized, whereas the coupling between the $a_{1} / a_{1}$, $a_{2} / a_{2}, a_{1} / a_{2}$, and $b_{1} / b_{1}$ terms is polarization preserving. With this rapid explosion of coupled scattering amplitudes it seems preferable ${ }^{14}$ to use the entire multiple Mie scattering formalism of Ref. 15 rather than treat the large coupled-amplitude system analytically.

\section{Order of the Scattering Solution of the Multiple-Scattering Equations}

As is derived below in Eqs. (30)-(34), the regime of beginning multiple Rayleigh scattering corresponds to

$$
3 f\left(k R_{\text {ave }}\right)(k a)^{3}\left[\left(n^{2}-1\right) /\left(n^{2}+2\right)\right]^{2} \lesssim 1.5 .
$$

In this regime, the iteration of Eqs. (17) and (18) converges, and the multiply scattered electric field arriving at the detector is a superposition of the fields scattered only a few times by the particles. The iteration solution of Eqs. (9), (15), (17), and (18) is called the order-of-scattering representation of the multiply scattered electric field. $15,16,25,26$ In a particular $p$-order multiple-scattering sequence, denoted here by $c_{p}$, light is scattered $p$ times by suspended particles before arriving at the detector: First by particle $n$, then by particle $m$, and so on until it is scattered penultimately by particle $\ell$ and lastly by particle $j$. Scattering by a given particle may occur a number of times within a scattering sequence. This situation is more likely for small particles for which single scattering is more nearly isotropic than for large particles for which single scattering is strongly forward peaked. The set of all $p$-order scattering sequences is denoted here by $\left\{c_{p}\right\}$.

The single-scattering (i.e., $p=1$ ) amplitudes of Eq. (17) are

$$
\left[\begin{array}{l}
P_{+} \\
P_{0} \\
P_{-}
\end{array}\right]^{(1)}=(3 / 2) a_{1} E_{0} \sum_{j=1}^{J} \exp \left[i\left(\mathbf{k}_{\text {inc }}-\mathbf{k}_{\text {scatt }}\right) \cdot \mathbf{r}_{j}\right]\left[\begin{array}{c}
b_{+} \\
b_{0} \\
b_{-}
\end{array}\right],
$$

and the multiple-scattering (i.e., $p \geq 2$ ) amplitudes are

$$
\begin{aligned}
{\left[\begin{array}{c}
P_{+} \\
P_{0} \\
P_{-}
\end{array}\right]^{(p)}=} & \left(3 a_{1} / 2\right)^{p}(i / k)^{p-1} E_{0} \\
& \times \sum_{\left\{C_{p}\right\}} \exp (i \Phi)\left(r_{n m} \ldots r_{\ell j}\right)^{-1} \\
& \times\left[T_{\ell j}\right] \ldots\left[T_{n m}\right]\left[\begin{array}{l}
b_{+} \\
b_{0} \\
b_{-}
\end{array}\right]
\end{aligned}
$$

where

$$
\Phi=\exp \left[i\left(\mathbf{k}_{\mathrm{inc}}-\mathbf{k}_{n m}\right) \cdot \mathbf{r}_{n}\right] \ldots \exp \left[i\left(\mathbf{k}_{\ell j}-\mathbf{k}_{\mathrm{scatt}}\right) \cdot \mathbf{r}_{j}\right] .
$$

The phase in Eq. (22) is a consequence of the time required by the light to traverse the scattering sequence $c_{p}$ before leaving the sample cell. Substitution of Eqs. (21) and (22) into Eqs. (15) gives the polarization-resolved $p$-order multiply scattered electric field

$$
\begin{aligned}
& E_{\alpha \beta}^{\mathrm{total}(p)}(\mathbf{r})=\left[E_{0} \exp (i k r) / r\right]\left[\left(n^{2}-1\right) k^{2} a^{3} /\left(n^{2}+2\right)\right]^{p} \\
& \quad \times \sum_{\left\{C_{p}\right\}} \exp (i \Phi) W_{\alpha \beta}^{(p)}\left(\theta, \Omega_{n m}, \ldots, \Omega_{\ell j}\right) /\left(r_{n m} \ldots r_{\ell j}\right),
\end{aligned}
$$

where $\alpha=V$ or $\alpha=H$ for the incoming plane wave, $\beta=V$ or $\beta=H$ for the multiply scattered light reaching the detector, and $\Omega_{\ell j}$ is the direction of particle $\ell$ with respect to particle $j$. The weighting factor $W_{\alpha \beta}^{(p)}$ for the scattering sequence $c_{p}$ gives the amplitude of the light that multiply scatters through the sample cell while taking the path from particle $n$ to particle $m$, and so on, to particle $\ell$ to particle $j$ to the detector. This weighting factor depends on both the polarization channel $\alpha \beta$ and the scattering angle 
$\theta$ in addition to the scattering sequence $c_{p}$. The weighting factor $W_{\alpha \beta}^{(p)}$ is calculated for each scattering sequence by one's multiplying together the appropriate number of $T$ matrices in Eq. (21) and putting in the angle dependence with Eqs. (15). The specific form for $W_{\alpha \beta}^{(p)}$ for $p=2,3$ is given in Appendix A. Also, there are no Beer's law beam-attenuation factors between the $p$ scatterings in Eq. (23). These factors arise as an interference effect in the coherent superposition of the various $p$-order forwardscattered fields. ${ }^{29}$

\section{E. Isotropic Scattering of Scalar Waves}

To assess the importance of both the polarizationchannel and the scattering-angle dependence of the single-scattering intensity in multiple Rayleigh scattering, we also examine multiple scattering of scalar waves by a collection of identical spherical particles. ${ }^{17}$ Because scalar waves are without polarization, the electric field vector in Eqs. (1)-(3) is replaced by the scalar wave amplitude $\psi$. In analogy to Mie theory, one determines the amplitude of the scattered partial waves by matching the boundary conditions for $\psi$ and its normal derivative for the incident, the scattered, and the interior waves at the surface of the particle. In the long-wavelength limit, $k a \ll 1$, the partialwave sum for the scattered wave contains only the isotropic scattering $L=0$ term and is called $s$-wave scattering. For multiple $s$-wave scattering of an incoming plane wave of amplitude $\psi_{0}$ by a collection of $J$ particles Eqs. (7)-(10) become

$$
\psi^{\text {total }}(\mathbf{r})=[i \exp (i k r) /(k r)] \sum_{j=1}^{J} \psi_{j} \exp \left(-i \mathbf{k}_{\text {scatt }} \cdot \mathbf{r}_{j}\right),
$$

with

$$
\psi_{j}=\psi_{0} a_{0} \exp \left(i \mathbf{k}_{\mathrm{inc}} \cdot \mathbf{r}_{j}\right)+i a_{0} \sum_{\substack{\ell=1 \\ \ell \neq j}}^{J} \psi_{\ell} \exp \left(i k r_{\ell j}\right) /\left(k r_{\ell j}\right) .
$$

In analogy to the Rayleigh scattering amplitude $a_{1}$ in Mie theory the $L=0$ scattering amplitude for scalar wave scattering in the $k a \ll 1$ limit is found to be

$$
a_{0}=-(i / 3)\left(n^{2}-1\right)(k a)^{3},
$$

where $n$ is the ratio of the wave number of the scalar wave inside the particle to that in the surrounding medium. Solving Eqs. (24) and (25) by iteration, one obtains

$$
\begin{aligned}
\psi^{(p) \text { total }}(\mathbf{r})= & {\left[\psi_{0} \exp (i k r) / r\right]\left(i a_{0} / k\right)^{p} } \\
& \times \sum_{\left\{C_{p}\right\}} \exp (i \Phi) /\left(r_{n m} \ldots r_{\ell j}\right) .
\end{aligned}
$$

Equation (27) is identical to Eq. (23) except for the absence of the scattering-sequence weighting factor $W_{\alpha \beta}^{(p)}$ that contained the polarization-channel and the scattering-angle dependence of electromagnetic Rayleigh scattering.

\section{Ensemble-Averaged Autocorrelation Function}

\section{A. Electromagnetic Waves}

In this subsection the incoherent portion of the electric field autocorrelation function is constructed from the $p$-order multiply scattered fields and is then ensemble averaged, scattering order by scattering order. The particles' positions in the scattering cell are assumed to be random, and their motion in the liquid is diffusive. As a result, given that particle $j$ is at position $\mathbf{r}_{j}$ at time $t$, the probability that it has moved to position $\mathbf{r}_{j}+\Delta$ at time $t+\tau$ is

$$
P_{j}(\Delta)=\exp \left(-\Delta^{2} / 4 D \tau\right) /(4 \pi D \tau)^{3 / 2},
$$

where $D$ is the diffusion coefficient of the particles in the liquid. The field autocorrelation function at time $t$ is defined as

$$
g_{\alpha \beta}(t, \tau, \theta)=\left|\mathbf{E}_{\alpha \beta} *(t+\tau, \theta) \times \mathbf{B}_{\alpha \beta}(t, \theta)\right| /\left(2 \mu_{0} c\right),
$$

where $\tau$ is the autocorrelation decay time and $\mu_{0}$ is the permeability of free space. The $\tau=0$ limit of Eq. (29) is the intensity of the multiply scattered light reaching the detector at time $t$. Substituting Eq. (23) and the analogous expression for the multiply scattered magnetic field into Eq. (29) shows that the result contains (i) products of the fields for the different scattering orders $p$ and $p^{\prime}$ and (ii) products of the fields for the same scattering order $p$. The type (ii) terms contain (a) products of fields for the two different scattering sequences $c_{p}$ and $c_{p}{ }^{\prime}$ and (b) products of the fields for the same scattering sequence $c_{p}$. The incoherent portion of the field autocorrelation function of Eq. (29) includes only type (ii) (b) terms and is a good approximation to the total autocorrelation function for beginning multiple scattering in which the volume fraction of particles in suspension is small. 2,5

The usual electric field autocorrelation function $g_{\alpha \beta}(\tau, \theta)$ is obtained by one's averaging Eq. (29) over all times $t$, which is equivalent ${ }^{17}$ to ensembleaveraging the positions of all the particles at times $t$ and $t+\tau$. The sums over particles at $t$ are converted to integrals over particle positions weighted by the particle number density, and the sums over particles at $t+\tau$ are converted to integrals over particle displacements $\Delta_{j}$ weighted by the probability factor of Eq. (28). As a result, the ensemble average of the $p$-order multiple-scattering autocorrelation function requires that $6 p$ integrals over particle positions be performed. Integration over $\Delta_{j}$ gives rise to the scattering-structure factors $\exp \left(-K_{j}^{2} D \tau\right)$, where $\mathbf{K}_{j}$ is the momentum transfer of the light scattered by particle $j$, leaving $3 p$ integrals to be performed. Integration over the particle positions at $t$ can be converted into integration over the position of particle $j$, the position of particle $\ell$ with respect to particle $j$, and so on, ending with the position of particle $n$ with respect to particle $m$. The integral over $\mathbf{r}_{j}$ is 
simply evaluated, leaving $3 p-3$ integrals to be performed. As was mentioned above, it is assumed that each particle in the sample cell receives light emitted by a random distribution of other particles out to a radius $R_{\text {ave }}$. This assumption allows the $p-1$ radial integrals over relative coordinates to be performed simply, leaving $2 p-2$ integrals over relative angular coordinates. The details of the ensemble averaging are carried out in Appendix A. The electric field autocorrelation function then becomes

$$
g_{\alpha \beta}(\tau, \theta)=J I_{V V}^{\mathrm{each}} \sum_{p=1}^{\infty} Q^{p-1} g_{\alpha \beta}^{(p)}(\tau, \theta),
$$

where $I_{V V}^{\text {each }}$ is the single-scattered intensity of each particle in the $V V$ polarization channel,

$$
g_{\alpha \beta}^{(1)}(\tau, \theta)=\left[W_{\alpha \beta}^{(1)}(\theta)\right]^{2} \exp \left(-\left|\mathbf{k}_{\text {inc }}-\mathbf{k}_{\text {scatt }}\right|^{2} D \tau\right)
$$

for single scattering $(p=1)$ with

$$
W_{V V}^{(1)}=1, \quad W_{V H}^{(1)}=W_{H V}^{(1)}=0, \quad W_{H H}^{(1)}=\cos ^{2} \theta,
$$

and

$$
\begin{aligned}
g_{\alpha \beta}^{(p)}(\tau, \theta)= & (4 \pi)^{p-1} \int \mathrm{d} \Omega_{\ell j} . . \mathrm{d} \Omega_{n m} \\
& \times\left[W_{\alpha \beta}^{(p)}\left(\theta, \Omega_{n m}, \ldots, \Omega_{\ell j}\right)\right]^{2} \\
& \times \exp \left(-\left|\mathbf{k}_{\mathrm{inc}}-\mathbf{k}_{n m}\right|^{2} D \tau\right) \ldots \\
& \times \exp \left(-\left|\mathbf{k}_{\ell j}-\mathbf{k}_{\text {scatt }}\right|^{2} D \tau\right)
\end{aligned}
$$

for higher-order scattering $(p \geq 2)$ with

$$
Q=3 f\left(k R_{\text {ave }}\right)(k a)^{3}\left[\left(n^{2}-1\right) /\left(n^{2}+2\right)\right]^{2} .
$$

For a typical dynamic light-scattering experiment ${ }^{30}$ with $\lambda=0.5145 \mu \mathrm{m}, a=0.05 \mu \mathrm{m}, R_{\text {ave }} \approx 2 \mathrm{~mm}, m=$ 1.333 , and $n=1.2$ corresponding to polystyrene latex (PSL) spheres in water, Eq. (34) becomes $Q \approx 860 f$. As a result, the regime of beginning multiple scattering corresponds to a volume fraction of suspended particles of $f \lesssim 1.7 \times 10^{-3}$.

\section{B. Scalar Waves}

Following a similar procedure, one finds that the incoherent portion of the ensemble-averaged autocorrelation function for multiple $s$-wave scattering of scalar waves, denoted hereafter by the subscript $I$, becomes

$$
g_{I}(\tau, \theta)=J I^{\text {each }} \sum_{p=1}^{\infty}\left(Q^{\prime}\right)^{p-1} g_{I}^{(p)}(\tau, \theta)
$$

with

$$
g_{I}^{(1)}(\tau, \theta)=\exp \left(-\left|\mathbf{k}_{\text {inc }}-\mathbf{k}_{\text {scatt }}\right|^{2} D \tau\right)
$$

for single scattering $(p=1)$ and

$$
\begin{aligned}
g_{I}^{(p)}(\tau, \theta)= & (4 \pi)^{p-1} \int \mathrm{d} \Omega_{\ell j} \ldots \mathrm{d} \Omega_{n m} \\
& \times \exp \left(-\left|\mathbf{k}_{\text {inc }}-\mathbf{k}_{n m}\right|^{2} D \tau\right) \ldots \\
& \times \exp \left(-\left|\mathbf{k}_{\ell j}-\mathbf{k}_{\text {scatt }}\right|^{2} D \tau\right)
\end{aligned}
$$

for higher-order scattering $(p \geq 2)$ with

$$
Q^{\prime}=f\left(k R_{\text {ave }}\right)(k a)^{3}\left(n^{2}-1\right)^{2} / 3 .
$$

The autocorrelation functions of Eqs. (36) and (37) have the $\tau=0$ value of

$$
g_{I}^{(p)}(0, \theta)=1 .
$$

Equations (30)-(34) for electromagnetic multiple scattering and Eqs. (35)-(38) for scalar wave multiple scattering contain identical scattering-structure factors because it was assumed in each case that the motion of the suspended particles is diffusive. But, as was mentioned after Eq. (27), the polarizationchannel- and the scattering-angle-dependent factor $\left[W_{\alpha \beta}^{(p)}\right]^{2}$ that weighted each sequence of electromagnetic scatterings in Eq. (33) is absent in Eq. (37) for scalar wave multiple scattering. It should also be noted that, even though $s$-wave single scattering of scalar waves is isotropic, multiple $s$-wave scattering is anisotropic as a result of the scattering-angle dependence of the scattering-structure factors in Eq. (37).

\section{Polarization-Resolved Multiply Scattered Intensity}

At $\tau=0$, only the scattering-sequence weighting factor is present in the integrand of Eq. (33), and Eqs. (30)-(34) reduce to the $p$-order scattered intensity. If the notation

$$
I_{\alpha \beta}^{(p)}(\theta) \equiv g_{\alpha \beta}^{(p)}(0, \theta)
$$

is used, Eq. (33) can be integrated analytically, after much algebra, to yield

$$
\begin{aligned}
& I_{V V}^{(p)}(\theta)=(1 / 15)^{p-1} A_{p}, \\
& I_{V H}^{(p)}(\theta)=I_{H V}^{(p)}(\theta)=(1 / 15)^{p-1} B_{p}, \\
& I_{H H}^{(p)}(\theta)=(1 / 15)^{p-1}\left[\left(A_{p}-B_{p}\right) \cos ^{2} \theta+B_{p}\right],
\end{aligned}
$$

where

$$
A_{1}=1, \quad B_{1}=0
$$

for single scattering, and $A_{p}$ and $B_{p}$ for $p \geq 2$ are given by the recursion relations

$$
\begin{aligned}
& A_{p}=8 A_{p-1}+2 B_{p-1}, \\
& B_{p}=A_{p-1}+9 B_{p-1} .
\end{aligned}
$$

As can be seen from Eqs. (41) and (42), the $V V$ single-scattering intensity is independent of $\theta$ in the $x z$ plane, the $V H$ and the $H V$ intensities vanish, and the $H H$ intensity is proportional to $\cos ^{2} \theta$. For increasing $p$, the homogenizing effect of multiple scattering gradually decreases the polarization-channel ${ }^{31}$ 
and the scattering-angle dependence of the $p$-order multiple-scattering intensity. Specifically, iteration of Eqs. (43) gives

$$
\begin{aligned}
& I_{V V}^{(2)}=(8 / 15), \\
& I_{V V}^{(4)}=1.098(8 / 15)^{3}, \\
& I_{V V}^{(6)}=1.359(8 / 15)^{5}, \\
& I_{V V}^{(8)}=1.852(8 / 15)^{7}, \\
& I_{V V}^{(10)}=2.685(8 / 15)^{9}
\end{aligned}
$$

for the even- $p$ intensity contributions to $V V$ multiple scattering,

$$
\begin{aligned}
& I_{V H}^{(2)}=0.125 I_{V V}^{(2)}, \\
& I_{V H}^{(4)}=0.390 I_{V V}^{(4)}, \\
& I_{V H}^{(6)}=0.623 I_{V V}^{(6)}, \\
& I_{V H}^{(8)}=0.788 I_{V V}^{(8)}, \\
& I_{V H}^{(10)}=0.888 I_{V V}^{(10)}
\end{aligned}
$$

for the even-p intensity contributions to $V H$ and $H V$ multiple scattering, and

$$
\begin{aligned}
& I_{H H}^{(2)}=\left(0.125+0.875 \cos ^{2} \theta\right) I_{V V}^{(2)}, \\
& I_{H H}^{(4)}=\left(0.390+0.610 \cos ^{2} \theta\right) I_{V V}^{(4)}, \\
& I_{H H}^{(6)}=\left(0.623+0.377 \cos ^{2} \theta\right) I_{V V}^{(6)}, \\
& I_{H H}^{(8)}=\left(0.788+0.212 \cos ^{2} \theta\right) I_{V V}^{(8)}, \\
& I_{H H}^{(10)}=\left(0.888+0.112 \cos ^{2} \theta\right) I_{V V}^{(10)}
\end{aligned}
$$

for the $H H$ polarization. More than 10 scatterings are required to eliminate the polarization and the angle dependence of multiple Rayleigh scattering. The $V H$ intensity for $p=2,3$ agrees with the $p=2$, 3 depolarization ratio obtained in Ref. 6, and Eqs. (41)-(43) generalize it to all scattering orders.

\section{Dependence of the Autocorrelation Function on the Delay Time}

A. Electromagnetic Multiple Scattering

For single scattering the polarization-resolved autocorrelation functions are exponential in the delay time $\tau$

$$
\begin{aligned}
& g_{V V}^{(1)}(\tau, \theta)=\exp \left[-2 T \sin ^{2}(\theta / 2)\right] \\
& g_{V H}^{(1)}(\tau, \theta)=g_{H V}^{(1)}(\tau, \theta)=0, \\
& g_{H H}^{(1)}(\tau, \theta)=\cos ^{2} \theta \exp \left[-2 T \sin ^{2}(\theta / 2)\right],
\end{aligned}
$$

where

$$
T=2 k^{2} D \tau .
$$

As was described in Ref. 10, the autocorrelation function of Eq. (33) for double scattering can be evaluated analytically for the idealized scattering geometry of Subsection 2.A in an $x^{\prime}, y^{\prime}, z^{\prime}$ coordinate system that is rotated with respect to the laboratory coordinate system of Fig. 1 by an angle $\theta / 2$ about the $y$ axis. In the rotated coordinate system the angle $\phi_{\ell j}{ }^{\prime}$ does not appear in the scattering-structure factor, thus greatly simplifying the integrals. The doublescattering autocorrelation function was found in Ref. 10 to be

$$
\begin{aligned}
g_{V V}^{(2)}(\tau, \theta)= & \exp (-2 T) \\
& \times\left[(3 / 8) F_{0}(\tau, \theta)+(1 / 4) F_{2}(\tau, \theta)\right. \\
& \left.+(3 / 8) F_{4}(\tau, \theta)\right], \\
g_{V H}^{(2)}(\tau, \theta)= & g_{H V}^{(2)}(\tau, \theta)=\exp (-2 T) \\
& \times\left\{\left[(1 / 8) \cos ^{2}(\theta / 2)\right] F_{0}(\tau, \theta)\right. \\
& +\left[-(3 / 4) \cos ^{2}(\theta / 2)+(1 / 2)\right] F_{2}(\tau, \theta) \\
& \left.+\left[(5 / 8) \cos ^{2}(\theta / 2)-(1 / 2)\right] F_{4}(\tau, \theta)\right\} \\
g_{H H}^{(2)}(\tau, \theta)= & \exp (-2 T) \\
& \times\left\{\left[(19 / 8) \cos ^{4}(\theta / 2)-3 \cos ^{2}(\theta / 2)\right.\right. \\
& +1] F_{0}(\tau, \theta)+\left[(-7 / 4) \cos ^{4}(\theta / 2)\right. \\
& \left.+4 \cos ^{2}(\theta / 2)-2\right] F_{2}(\tau, \theta) \\
& +\left[(3 / 8) \cos ^{4}(\theta / 2)-\cos ^{2}(\theta / 2)\right. \\
& \left.+1] F_{4}(\tau, \theta)\right\},
\end{aligned}
$$

where

$$
\begin{aligned}
F_{0}(\tau, \theta)= & (1 / 2) \int_{-1}^{1} \mathrm{~d} u \exp [2 T u \cos (\theta / 2)] \\
= & \sinh (s) / s \\
= & \sum_{\operatorname{even} n} s^{n} /[n !(n+1)], \\
F_{2}(\tau, \theta)= & (1 / 2) \int_{-1}^{1} \mathrm{~d} u u^{2} \exp [2 T u \cos (\theta / 2)] \\
= & \sinh (s) / s-2 \cosh (s) / s^{2}+2 \sinh (s) / s^{3} \\
= & \sum_{\operatorname{even} n} s^{n} /[n !(n+3)], \\
F_{4}(\tau, \theta)= & (1 / 2) \int_{-1}^{1} \mathrm{~d} u u^{4} \exp [2 T u \cos (\theta / 2)] \\
= & \sinh (s) / s-4 \cosh (s) / s^{2}+12 \sinh (s) / s^{3} \\
& -24 \cosh (s) / s^{4}+24 \sinh (s) / s^{5} \\
= & \sum_{\operatorname{even} n} s^{n} /[n !(n+5)],
\end{aligned}
$$

with

$$
s=2 T \cos (\theta / 2) .
$$

The hyperbolic function representation of Eqs. (50)(52) is most convenient for numerical computations when $s \geq 1$, and the series representation is most convenient when $s \lessgtr 1$.

The normalized $p$-order autocorrelation functions $g_{\alpha \beta}^{N(p)}(\tau, \theta)$ are defined by

$$
g_{\alpha \beta}^{(p)}(\tau, \theta)=I_{\alpha \beta}^{(p)}(\theta) g_{\alpha \beta}^{N(p)}(\tau, \theta)
$$



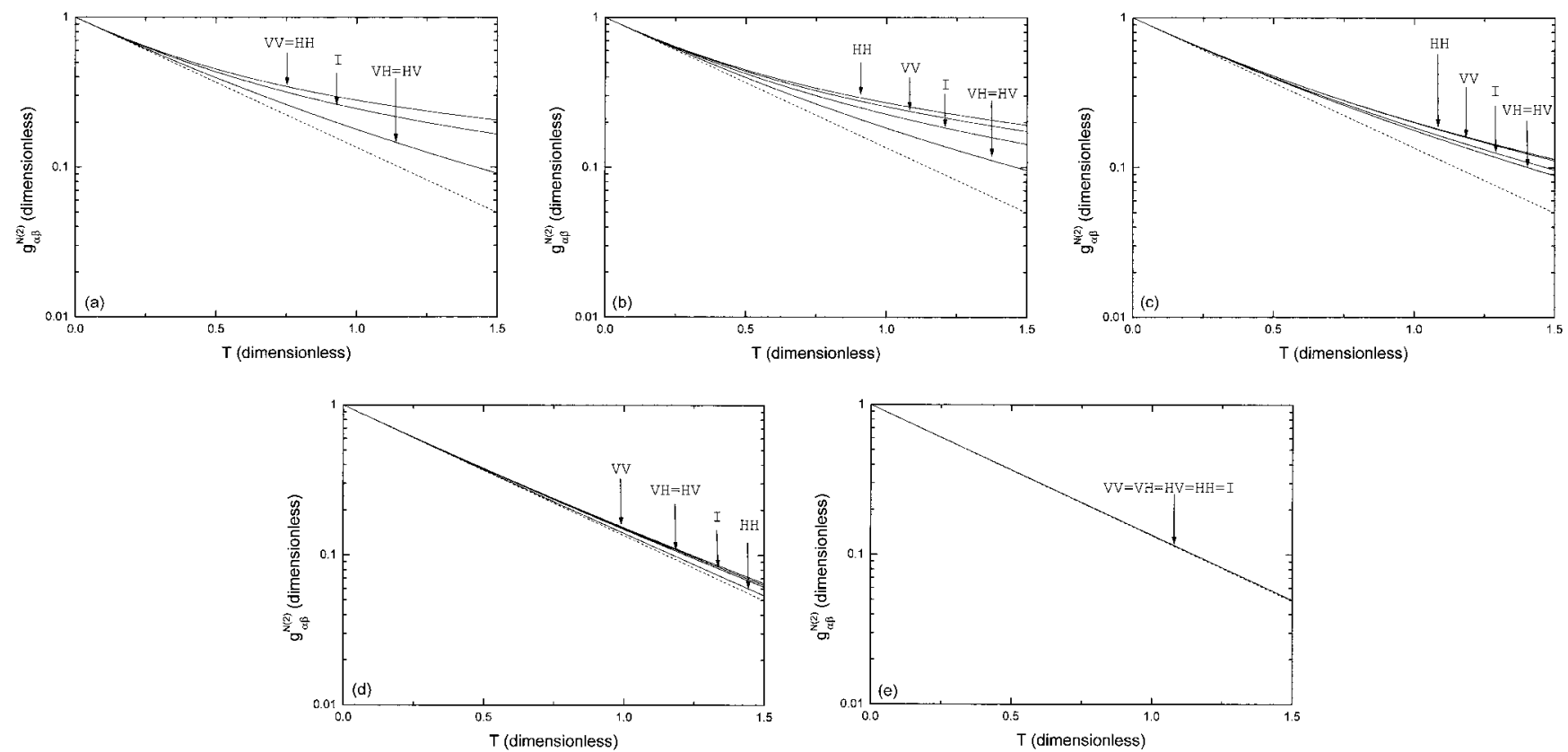

Fig. 2. Normalized $p=2$ autocorrelation function plotted as a function of the scaled delay time $T$ for scattering angles of (a) $0^{\circ}$, (b) $45^{\circ}$, (c) $90^{\circ}$, (d) $135^{\circ}$, (e) $180^{\circ}$ for $V V, V H, H V$, and $H H$ scattering and for multiple $s$-wave scattering of scalar waves, denoted $I$. The dashed curves represent the function $\exp (-2 T)$.

so that

$$
g_{\alpha \beta}^{N(p)}(0, \theta)=1
$$

and are hereafter denoted by the superscript $N$. The double-scattering, $p=2$, normalized autocorrelation functions of Eqs. (49)-(53) are graphed in Figs. 2(a)$2(\mathrm{e})$. Both functions are nonexponential in $\tau$ and polarization-channel dependent, except at $\theta=180^{\circ}$.
The decaying exponential $\exp (-2 T)$ is given by the dashed curves in the graphs. Although a rotation of coordinates permitted the double-scattering autocorrelation functions to be evaluated analytically, a similar rotation appears not to provide any real simplification for third-order scattering and beyond because the algebraic complexity of the scatteringsequence weighting factor when expressed in the
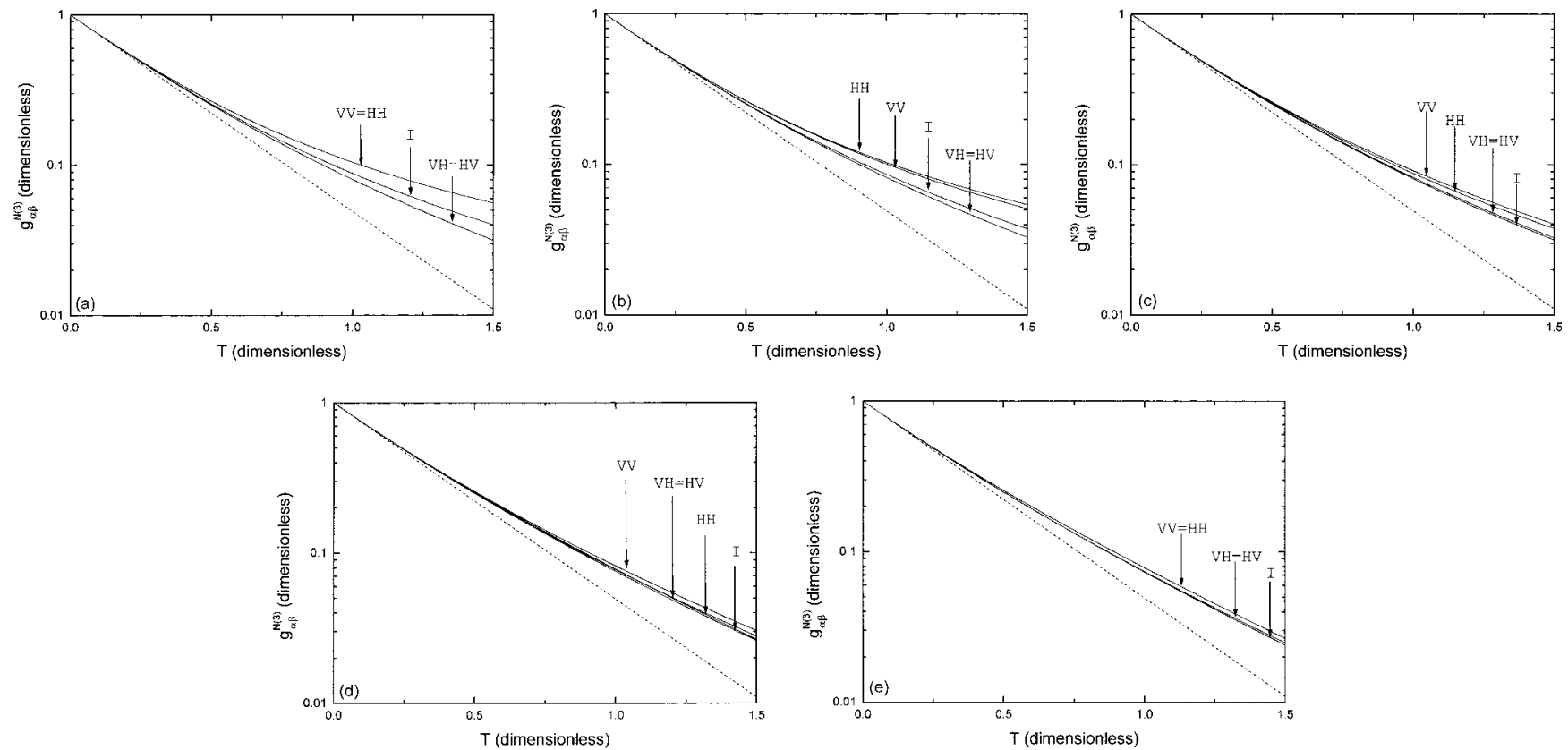

Fig. 3. Normalized $p=3$ autocorrelation function plotted as a function of the scaled delay time $T$ for scattering angles of (a) $0^{\circ}$, (b) $45^{\circ}$, (c) $90^{\circ}$, (d) $135^{\circ}$, (e) $180^{\circ}$ for $V V, V H, H V$, and $H H$ scattering and for multiple $s$-wave scattering of scalar waves. The dashed curves represent the function $\exp (-3 T)$. 

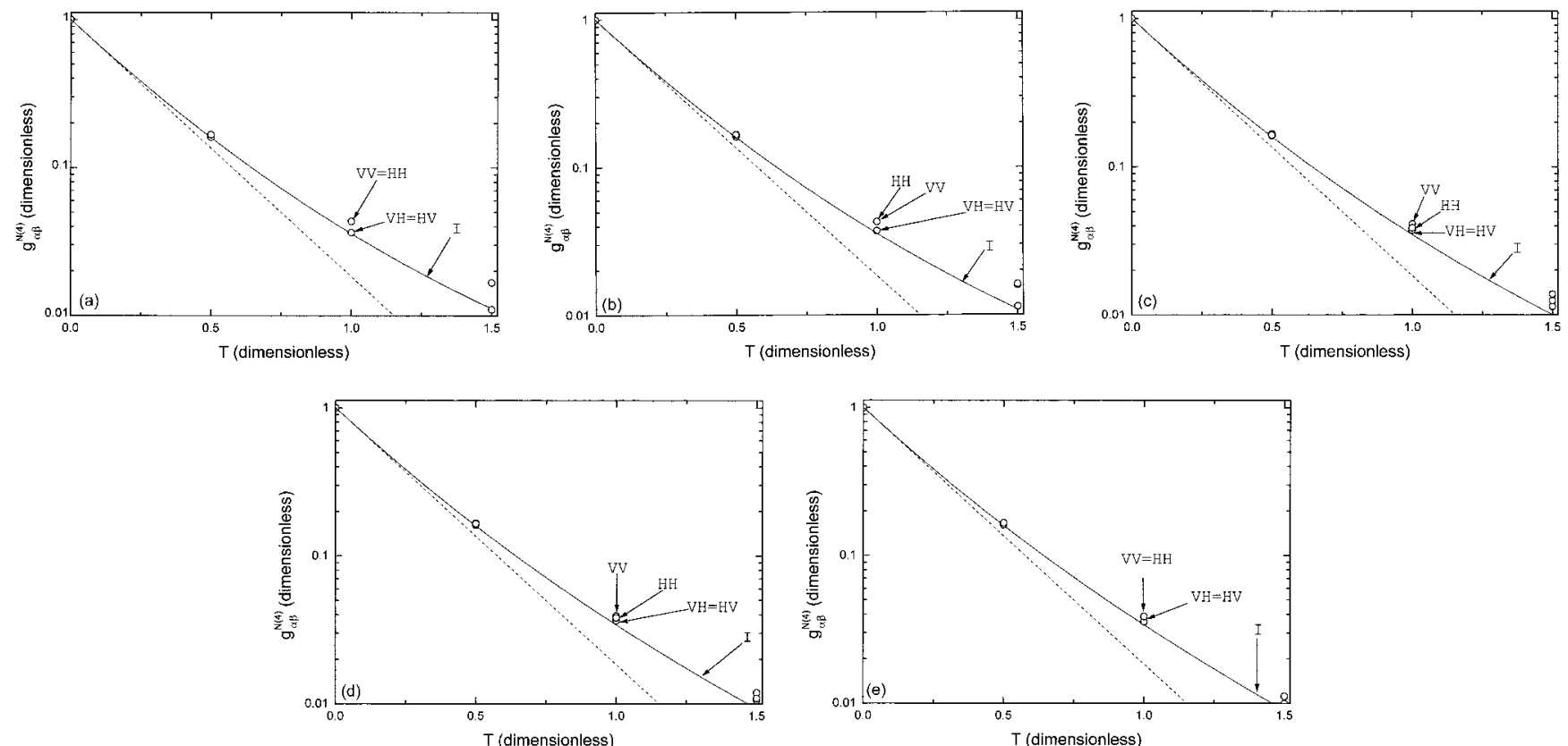

Fig. 4. Normalized $p=4$ autocorrelation function plotted as a function of the scaled delay time $T$ for scattering angles of (a) $0^{\circ}$, (b) $45^{\circ}$, (c) $90^{\circ}$, (d) $135^{\circ}$, (e) $180^{\circ}$ for $V V, V H, H V$, and $H H$ scattering (open circles) and for multiple $s$-wave scattering of scalar waves (solid curve). The dashed curves represent the function $\exp (-4 T)$.

rotated-coordinate system offsets the advantage of the analytic integration.

The normalized triple-scattering, $p=3$, and quadruple-scattering, $p=4$, autocorrelation functions of Eqs. (33) and (54) were evaluated for $\theta=0^{\circ}$, $45^{\circ}, 90^{\circ}, 135^{\circ}, 180^{\circ}$ and for $0 \leq T \leq 1.5$ by one's numerically performing the fourfold and the sixfold integrals over the relative angular coordinates. The $p=4$ calculations were performed for only a few values of $T$ because of the computational burden involved. The results are shown in Figs. 3(a)-3(e) and 4(a)-4(e), respectively, in which the decaying exponentials $\exp (-3 T)$ and $\exp (-4 T)$, respectively, are given by the dashed curves. The $p$-order normalized autocorrelation functions exhibit a rapidly decreasing polarization-channel dependence as the order of scattering increases, and the dependence is minimal for $p \gtrsim 3$ and $\theta \gtrsim 90^{\circ}$. This trend is contrasted with the behavior of the $p$-order-scattering normalization factor $I_{\alpha \beta}^{(p)}$ of Section 4 for which more than 10 scatterings were required to erase the memory of the single-scattering polarization. Thus the $p$-order autocorrelation function of Eq. (54) contains a factor that possesses only weak polarization dependence multiplied by another factor that possesses the majority of the polarization dependence.

It was also found that $g_{V H}^{N(p)}(\tau, \theta)=g_{H V}^{N(p)}(\tau, \theta)$ for $p=$ $1,2,3,4$. It is conjectured that this relation is true in general for the idealized geometry of Subsection 2.A. Experimental polarization-resolved autocorrelation data on suspensions ${ }^{8}$ of $a=0.044 \mu \mathrm{m}$ (i.e., $k a=0.80$ ) and $a=0.210 \mu \mathrm{m}$ (i.e., $k a=4.04$ ) particles in decane and toluene, respectively, indicate that $g_{V H}(\tau, \theta)$ and $g_{H V}(\tau, \theta)$ are nearly equal, especially for $\theta \gtrsim 80^{\circ}$. This result is consistent with the data shown in Figs. 2-4 and gives encouragement that the approximate autocorrelation function derived here for the idealized geometry and the Rayleigh scatterers remains approximately valid for more realistic experimental dynamic light-scattering geometries and somewhat larger-size particles.

B. Multiple Scattering of Scalar Waves

For single $s$-wave scattering of scalar waves in the long-wavelength limit, one obtains

$$
g_{I}^{(1)}(\tau, \theta)=\exp \left[-2 T \sin ^{2}(\theta / 2)\right]
$$

For multiple scattering, $p \geq 2$, the autocorrelation function of Eq. (37) contains $2 p-2$ integrals over relative angular coordinates and may be further simplified by use of

$$
\int_{0}^{2 \pi} \mathrm{d} \phi_{\ell j} \exp \left(\gamma \cos \phi_{\ell j}\right)=2 \pi I_{0}(\gamma),
$$

where $I_{0}$ is a modified Bessel function, to evaluate all $p-1$ relative $\phi$ integrals, leaving $p-1$ integrals to be performed. Similarly,

$$
\begin{aligned}
\int_{0}^{2 \pi} \sin \theta_{\ell j} \mathrm{~d} \theta_{\ell j} \exp \left[B(1+\cos \theta) \cos \theta_{\ell j}\right] \\
\times I_{0}\left(B \sin \theta \sin \theta_{\ell j}\right) \\
=2 \sinh [2 B \cos (\theta / 2)] /[2 B \cos (\theta / 2)]
\end{aligned}
$$

can be used to evaluate the first relative $\theta$ integral in the scattering sequence, leaving only $p-2$ integrals 

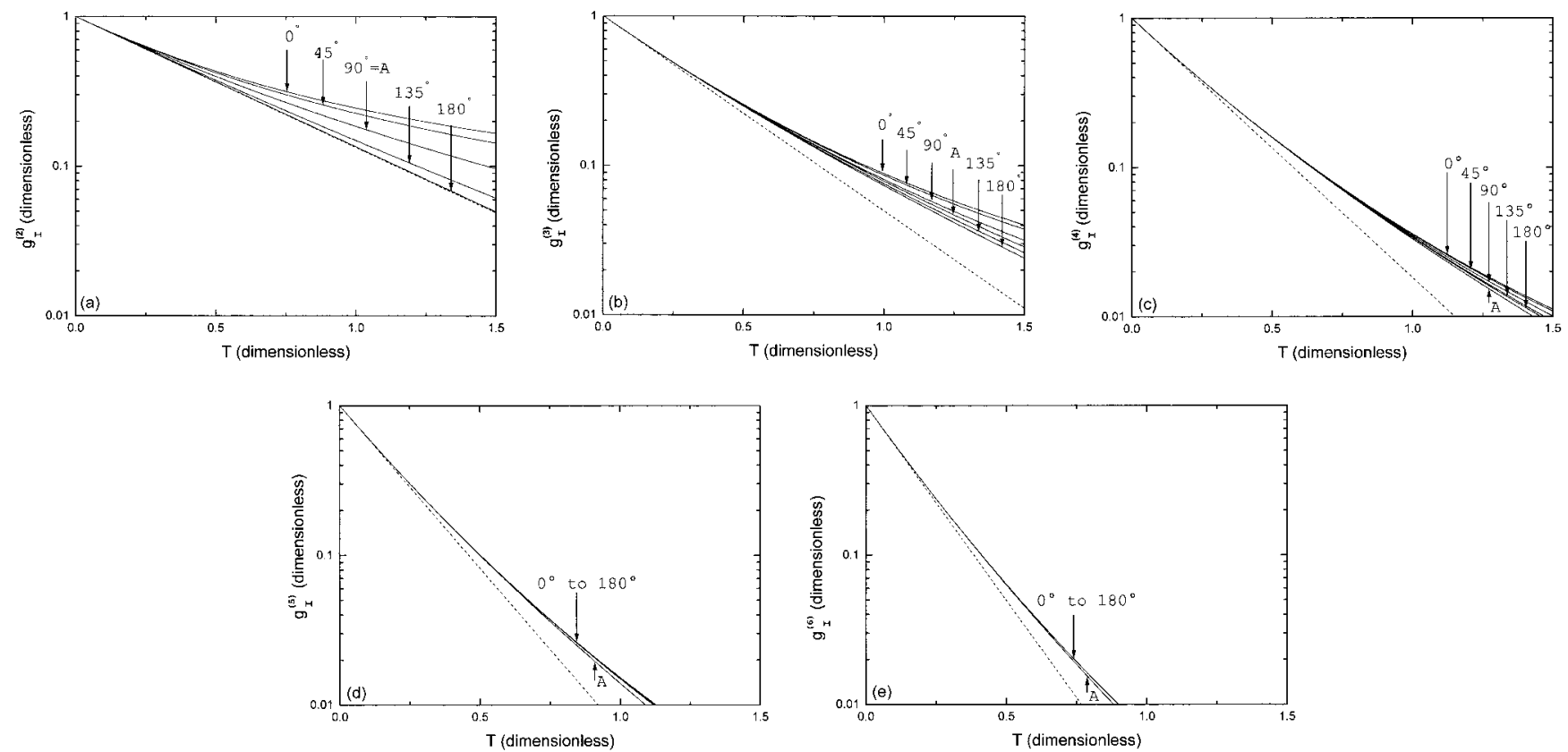

Fig. 5. Autocorrelation function for multiple $s$-wave scattering of scalar waves plotted as a function of the scaled delay time $T$ for (a) $p=$ 2, (b) $p=3$, (c) $p=4$, (d) $p=5$, (e) $p=6$ and for $\theta=0^{\circ}, 45^{\circ}, 90^{\circ}, 135^{\circ}, 180^{\circ}$. The approximation to Eq. (62) is denoted $A$, and the dashed curve represents the function $\exp (-p T)$.

to be performed numerically. For double scattering, one obtains

$$
\begin{aligned}
g_{I}^{(2)}(\tau, \theta)= & \exp (-2 T) \\
& \times \sinh [2 T \cos (\theta / 2)] /[2 T \cos (\theta / 2)],
\end{aligned}
$$

which, for comparison with the results for higherorder scattering described in Subsection 5.C, has the Taylor series expansion

$$
\begin{aligned}
g_{I}^{(2)}(\tau, \theta)= & \exp (-2 T)\left[1+(2 / 3) T^{2}\right. \\
& \left.-(2 / 3) T^{2} \sin ^{2}(\theta / 2)+\ldots\right] .
\end{aligned}
$$

The multiple $s$-wave scattering autocorrelation function for triple, quadruple, quintuple, and sextuple scattering was evaluated for $\theta=0^{\circ}, 45^{\circ}, 90^{\circ}, 135^{\circ}$, $180^{\circ}$ and for $0 \leq T \leq 1.5$ by one's numerically performing the single, twofold, threefold, and fourfold relative $\theta$ integrals. In Figs. $2-4$ the results are graphed for $p=2,3,4$ along with the normalized multiple Rayleigh scattering autocorrelation function $g_{\alpha \beta}^{N(p)}(\tau, \theta)$. These figures indicate that $g_{I}^{(p)}(\tau, \theta)$ is a good approximation to $g_{\alpha \beta}^{N(p)}(\tau, \theta)$, regardless of the polarization channel for $\theta \gtrsim 90^{\circ}$ when $p \gtrsim 3$. The multiple s-wave autocorrelation functions are also graphed in Figs. 5(a) -5 (e) for $2 \leq p \leq 6$. The decaying exponential $\exp (-p T)$ is given by the dashed curves in the graphs. These graphs indicate that $g_{I}^{(p)}(\tau, \theta)$ exhibits less $\theta$ dependence but remains nonexponential as $p$ increases and that the scatteringangle dependence is minimal for $p \gtrsim 3$. Again, this trend is contrasted with the behavior of the $p$-order normalization factor $I_{\alpha \beta}^{(p)}$, which requires more than 10 scatterings to erase the memory of the singlescattering anisotropy. As a result, only the normal- izing factor in Eq. (54) for the $p$-order autocorrelation function contains strong polarization-channel and scattering-angle dependence.

\section{Approximate Delay-Time Dependence of the $p$-Order Autocorrelation Function}

The $p$-order multiple $s$-wave scattering autocorrelation function was also calculated analytically for $p \geq$ 3 by Taylor series expansion of the integrand of Eq. (37) in powers of $T$ and integration term by term. After much algebra the result is

$$
\begin{aligned}
g_{I}^{(3)}(\tau, \theta)= & \exp (-3 T)\left[1+(3 / 6) T^{2}+(1 / 9) T^{3}\right. \\
& \left.-(2 / 9) T^{3} \sin ^{2}(\theta / 2)+\ldots\right], \\
g_{I}^{(4)}(\tau, \theta)= & \exp (-4 T)\left[1+(4 / 6) T^{2}+(32 / 135) T^{4}\right. \\
& \left.-(2 / 27) T^{4} \sin ^{2}(\theta / 2)+\ldots\right], \\
g_{I}^{(5)}(\tau, \theta)= & \exp (-5 T)\left[1+(5 / 6) T^{2}+\ldots\right] .
\end{aligned}
$$

The slope of the $p$-order autocorrelation function in Eqs. (60) and (61) at $T=0$ is equal to $-p$, in agreement with the result of Ref. 6 . The weakening of the scattering-angle dependence of $g_{I}^{(p)}(\tau, \theta)$ as the scattering order increases is evident in Eqs. (61) as the leading term in $\theta$ is proportional to $T^{p} / 3^{p-1}$. Equations (61) can be approximated by the function

$$
\begin{aligned}
g_{\text {approx }}^{(p)}(\tau)= & \exp (-p T) \sinh \left[(p)^{1 / 2} T\right] /\left[(p)^{1 / 2} T\right] \\
= & \exp (-p T)\left[1+(p / 6) T^{2}\right. \\
& \left.+\left(p^{2} / 120\right) T^{4}+\ldots\right] .
\end{aligned}
$$

Equations (61) and (62) are equal to the order $T^{2}$ and are nearly equal to the order $T^{4}$ when $\theta=90^{\circ}$. It should be noted that the approximate autocorrelation 
function of Eq. (62) contains no polarization-channel dependence or scattering-angle dependence; it is a function of the delay time alone.

The approximate autocorrelation function $g_{\text {approx }}^{(p)}(\tau)$, denoted by $A$, is graphed in Figs. 5(a) -5 (e) for $2 \leq p \leq$ 6 along with the multiple $s$-wave scattering autocorrelation function $g_{I}^{(p)}(\tau, \theta)$. Figure 5 illustrates that Eq. (62) provides a much better approximation to $g_{I}^{(p)}(\tau, \theta)$ than does the simple exponential $\exp (-p T)$, as was assumed in Refs. 2 and 6. For example, for $\theta=90^{\circ}$ and $T=1.0, g_{\text {approx }}^{(p)}$ differs from $g_{I}^{(p)}$ by $0.0021,0.0017$, 0.0012 , and 0.0007 for $p=3,4,5,6$, respectively, whereas $\exp (-p T)$ differs from $g_{I}^{(p)}$ by $0.0310,0.0166$, 0.0084 , and 0.0040 , respectively. Although the exponential approximation to $g_{I}^{(p)}(\tau, \theta)$ is not especially accurate for low orders of multiple scattering, it can be inferred from Figs. 5(a)-5(e) that it becomes adequate in the deep multiple-scattering regime of DWS where tens or hundreds of scatterings of light occur in the sample cell. One can summarize Figs. $2-5$ by saying that the polarization-channel insensitivity of $g_{\alpha \beta}^{N(p)}(\tau, \theta)$ for $p \gtrsim 3$ permitted it to be accurately approximated by $g_{I}^{(p)}(\tau, \theta)$ and that the $\theta$ insensitivity of $g_{I}^{(p)}(\tau, \theta)$ for $p \gtrsim$ 3 permitted it to be accurately approximated by $g_{\text {approx }}^{(p)}(\tau)$.

\section{Separable Model for the Polarization-Resolved Autocorrelation Function for Beginning Multiple Scattering}

The results of Sections 1-5 are now combined to provide a simple analytical model for the polarizationresolved multiple Rayleigh scattering electric field autocorrelation function $g_{\alpha \beta}(\tau, \theta)$. For beginning multiple scattering the iteration solution of the multiple-scattering equations converges. As a result, the incoherent portion of the autocorrelation function was written as a sum of contributions from each scattering order $p$. The $\tau$ dependence of the $p$-order autocorrelation function was found to approximately decouple from the polarization-channel and the scattering-angle dependence for triple scattering and beyond. The $\tau=0$ value of the $p$-order autocorrelation function contains most of the $\theta$ and the polarization dependence, whereas Eq. (62) gives an accurate approximation of the $\tau$ dependence. Combining these results, one obtains

$$
\begin{aligned}
g_{\alpha \beta}(\tau, \theta) \approx & J I_{\text {each }}\left[g_{\alpha \beta}^{(1)}(\tau, \theta)+Q g_{\alpha \beta}^{(2)}(\tau, \theta)\right. \\
& \left.+\sum_{p=3}^{\infty} Q^{p-1} I_{\alpha \beta}^{(p)}(\theta) g_{\text {approx }}^{(p)}(\tau)\right],
\end{aligned}
$$

with Eqs. (47) and (48) for the single-scattering autocorrelation function, Eqs. (49) for the doublescattering autocorrelation function, Eqs. (41)-(43) for the $p \geq 3$ scattered intensity, Eq. (62) for the $\tau$ dependence of the $p \geq 3$ normalized autocorrelation function, and Eq. (34) for the expansion parameter $Q$.

As a preliminary test of Eq. (63), its predictions were compared with experimental autocorrelation data taken at a scattering angle of $\theta=90^{\circ}$ for four samples of nominally $a=56 \mathrm{~nm}$ PSL spheres suspended in water in a 1.0-cm-diameter test tube at the center of an index-matching vat and illuminated with the $\lambda=0.5145 \mu \mathrm{m}$ beam of an $\mathrm{Ar}^{+}$laser. The test tube was filled with the PSL suspension to a height of approximately $1.0 \mathrm{~cm}$, making a roughly equidimensional sample. The scattered light was detected by a monomode optical fiber located at $r=20 \mathrm{~cm}$ from the center of the test tube and output to an avalanche photodiode that behaved as a wide-field-of-view detector. The incident beam was $V$ polarized, and the detected light was unpolarized. The samples had volume fractions of $f=1.7 \times 10^{-5}, 2.0 \times 10^{-4}, 1.0 \times$ $10^{-3}, 2.0 \times 10^{-3}$. The autocorrelation function of the first sample should be dominated by single scattering, and the other samples should be in the beginning multiple-scattering regime.

These experimental conditions violated a number of the assumptions on which Eq. (63) is based. The PSL sphere-size parameter is $k a=0.87$, which is somewhat beyond the Rayleigh limit. Only the PSL spheres within the beam volume were illuminated, rather than all the spheres in the sample cell. The model of Eq. (63) assumes a point detector, and the theoretical results should be integrated over the detector face. This last point is less of an issue for monomode fiber detectors whose core is much narrower than the spatial-coherence width of the scattered light. In addition, because the precise physical meaning of $R_{\text {ave }}$ is somewhat elusive, the point of view adopted here is that the data are considered successfully fitted by the model if the value of $R_{\text {ave }}$ obtained by the best fit is comparable with the smaller of either the radius of the test tube or the single-scattering mean free path, rather than being orders of magnitude larger or smaller.

The $f=1.7 \times 10^{-5}$ data were used to determine the particle size, assuming single scattering only. The single-scattering fit to the intensity autocorrelation function is shown in Fig. 6(a). The data were well fitted by $a=53.3 \mathrm{~nm}$ for $\tau \lesssim 0.3 \mathrm{~ms}$, in good agreement with the nominal $a=56 \mathrm{~nm}$ size quoted by the manufacturer of the PSL spheres. The data begin to rise above the fit for $\tau \gtrsim 0.4 \mathrm{~ms}$ because the incident beam was scattered only weakly by the low concentration of particles and accurate measurement of the autocorrelation function for long delay times was difficult. For comparison with Figs. 2-5, the delay time of $\tau=1.0 \mathrm{~ms}$ shown in Figs. 6(a)-6(d) corresponds to $T=2.173$ in Eq. (48).

Next, for the $f=2.0 \times 10^{-4}, 1.0 \times 10^{-3}, 2.0 \times 10^{-3}$ data and the particle size determined as shown in Fig. 6(a), the quantity $R_{\text {ave }}$ [and thus the quantity $Q$ related to it through Eq. (34)] was used as the only adjustable parameter in Eq. (63). The measured intensity autocorrelation function, with the baseline subtracted and normalized to unity at $\tau=0$, was modeled by the incoherent combination

$$
G^{N}(\tau, \theta)=\frac{\left[g_{V V}^{2}(\tau, \theta)+g_{V H}^{2}(\tau, \theta)\right]}{\left[g_{V V}^{2}(0, \theta)+g_{V H}^{2}(0, \theta)\right]} .
$$

The fit of Eqs. (63) and (64) to the data is shown in Figs. $6(\mathrm{~b})-6(\mathrm{~d})$. For $f=2.0 \times 10^{-4}, 1.0 \times 10^{-3}$, 

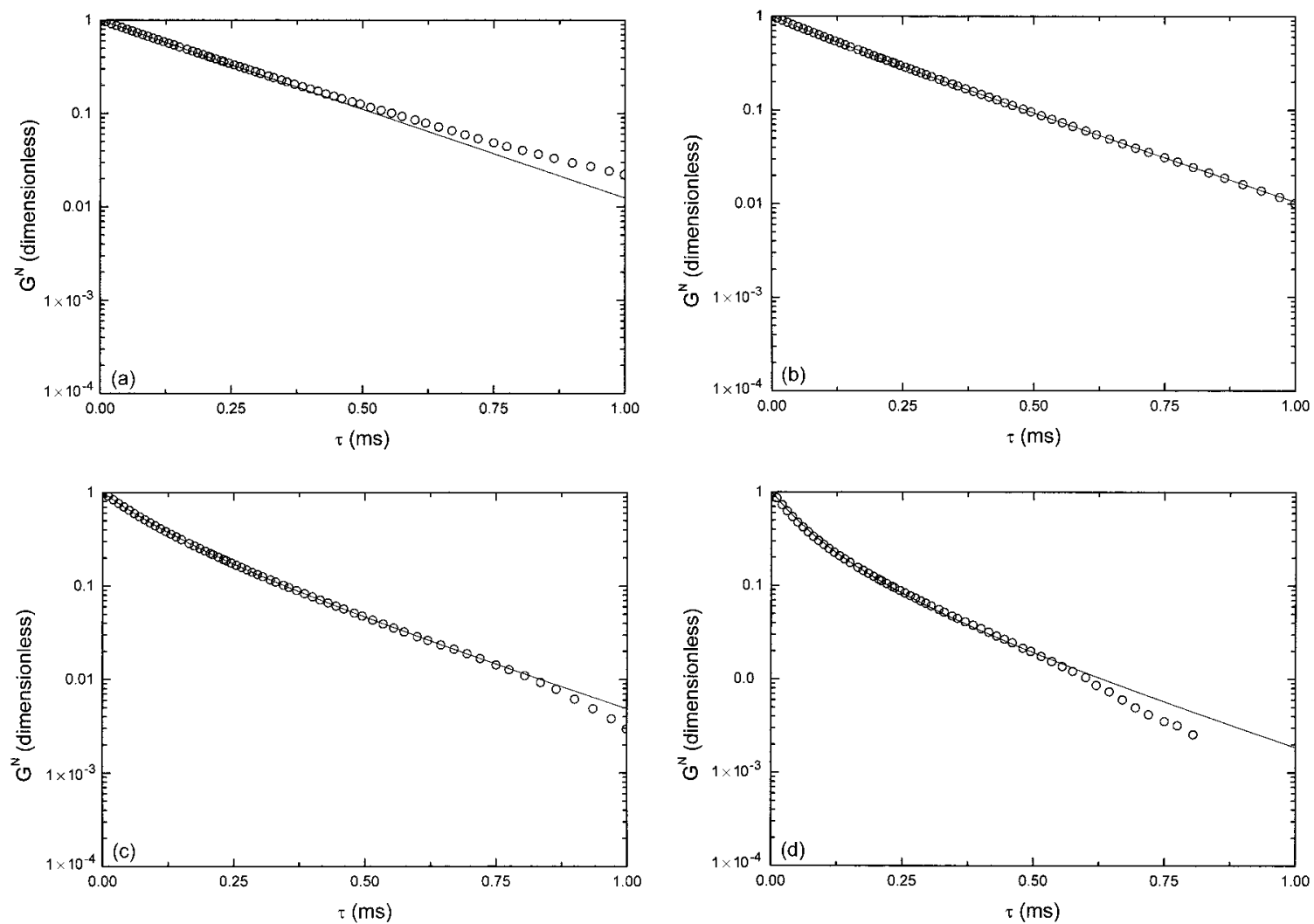

Fig. 6. Intensity autocorrelation function plotted as a function of the delay time $\tau$ for suspensions of $a=53.5 \mathrm{~nm}$ PSL spheres in water with volume fractions $f$ of (a) $1.7 \times 10^{-5}$, (b) $2.0 \times 10^{-4}$, (c) $1.0 \times 10^{-3}$, (d) $2.0 \times 10^{-3}$. The data are represented by the open circles, and the fit to Eqs. (63) and (64) by the solid curves. In (b)-(d) values of $R_{\text {ave }}=3.11,1.78,1.23 \mathrm{~mm}$ were used, respectively.

$2.0 \times 10^{-3}$ the data are well fitted by $R_{\text {ave }}=3.11$, $1.78,1.23 \mathrm{~mm}$, respectively. As was mentioned in Subsection 2.D, the $f=2.0 \times 10^{-3}$ data should be near the end of the regime of beginning multiple scattering. Because the higher concentrations of particles scatter light more strongly, the weak signal encountered in the data of Fig. 6(a) is not a concern. Rather, the fits deteriorate for $\tau \gtrsim 0.8 \mathrm{~ms}$ and $\tau \gtrsim 0.6$ $\mathrm{ms}$ in Figs. 6(c) and 6(d), respectively, owing to uncertainties in the experimental baseline subtraction. Pair-correlation effects and hydrodynamic interactions should not affect the measured autocorrelation functions until $f \gtrsim 0.01$, well above the concentrations examined here.

The parameters of the fits shown in Figs. 6(b)-6(d), the resulting percentages of various-order scattering in the $V V$ channel at $\tau=0$, and the single-scattering mean free path calculated by use of Mie theory are given in Table 1. Although the precise physical meaning of $R_{\text {ave }}$ is theoretically somewhat unclear, it is encouraging that our best-fit values of $R_{\text {ave }}$ are comparable with the smaller of either the sample-cell radius of $5.0 \mathrm{~mm}$ or the single-scattering mean free path. It is also encouraging that the model of Eq. (63) fits the data at all. If the model were woefully inaccurate, the shape of the autocorrelation function would not resemble the data for any value of $R_{\text {ave }}$, and even a best-fit value of $R_{\text {ave }}$ under these circumstances would provide a visually poor comparison when the data were graphed. This is not the case in Figs. 6(b)-6(d): The shape of the data is matched by the shape predicted by Eq. (63).

Much theoretical and experimental research on the beginning multiple-scattering regime remains. Experimentally, it is of interest to test the predictions of Eq. (63) with $V V, V H, H V$, and $H H$ polarizationresolved data. Further, it is of interest to determine

Table 1. Parameters of the Fit of the Multiple-Scattering Model of Eq. (63) to the Experimental Data for Suspensions of PSL Spheres in Water as Shown in Figs. 6(b)-6(d $)^{a}$

\begin{tabular}{llccc}
\hline & & \multicolumn{3}{c}{ Volume Fraction $f$} \\
\cline { 3 - 5 } \multicolumn{2}{c}{ Parameter } & $2.0 \times 10^{-4}$ & $1.0 \times 10^{-3}$ & $2.0 \times 10^{-3}$ \\
\hline \multicolumn{2}{c}{$L_{\text {single }}(\mathrm{mm})$} & 16.44 & 3.29 & 1.64 \\
$R_{\text {ave }}(\mathrm{mm})$ & 3.11 & 1.78 & 1.23 \\
$Q$ & & 0.325 & 0.929 & 1.281 \\
$p=1$ & $(\%)$ & 82.5 & 48.7 & 28 \\
$p=2$ & $(\%)$ & 14.3 & 24.2 & 20 \\
$p=3$ & $(\%)$ & 2.6 & 12.5 & 14 \\
$p=4$ & $(\%)$ & 0.5 & 6.5 & 10 \\
$p \geq 5$ & $(\%)$ & 0.1 & 8.1 & 28 \\
\hline
\end{tabular}

${ }^{a} L_{\text {single }}$ is the single-scattering mean free path obtained from Mie theory; $R_{\text {ave }}$ is obtained by the data-fitting procedure; $Q$ is obtained from $R_{\text {ave }}$ by use of Eq. (34); and $p=1,2,3,4$ and $p \geq 5$ denote the percentage of single-, double-, triple-, quadruple-, and quintupleand higher-order scattering in the $V V$ intensity at $\tau=0$ obtained from Eqs. (41)-(43) and (63). 
the range of particle sizes, cell geometries, and scattering angles for which the $V H$ and the $H V$ autocorrelation functions approximately coincide. To more closely approximate the idealized geometry considered here, one should expand the incident laser beam to illuminate the entire sample cell. Theoretically, it is of interest to use the full exact multiple-scattering formalism of Refs. 15, 16, and 21 to assess the effects of Mie corrections to multiple scattering. But most importantly from the theoretical side, the physical meaning of $R_{\text {ave }}$ needs to be elucidated. In spite of the large amount of investigation that yet remains, the comparison of the predictions of Eq. (63) with the experimen-
In addition, we obtain

$$
\begin{aligned}
& W_{H V}^{(2)}=-x_{\ell j} y_{\ell j} / r_{\ell j}^{2}, \\
& W_{H H}^{(2)}=D \cos (\theta)-C \sin (\theta),
\end{aligned}
$$

with

$$
\begin{aligned}
& C=-x_{\ell j} z_{\ell j} / r_{\ell j}{ }^{2}, \\
& D=\left(y_{\ell j}^{2}+z_{\ell j}{ }^{2}\right) / r_{\ell j}{ }^{2} .
\end{aligned}
$$

For triple scattering multiplication of the matrices $\left[T_{\ell j}\right]\left[T_{m \ell}\right]$ in Eqs. (15), (21), and (23) yields

$$
W_{W V}^{(3)}=\frac{\left[\left(x_{\ell j}^{2}+z_{\ell j}^{2}\right)\left(x_{m \ell}{ }^{2}+z_{m \ell}{ }^{2}\right)+\left(y_{\ell j} z_{\ell j}\right)\left(y_{m \ell} z_{m \ell}\right)+\left(x_{\ell j} y_{\ell j}\right)\left(x_{m \ell} y_{m \ell}\right)\right]}{\left(r_{\ell j}^{2} r_{m \ell}{ }^{2}\right)} .
$$

$W_{V H}^{(3)}$ is given by Eq. (A2) with

$$
\begin{aligned}
& A=\frac{\left[-\left(y_{\ell j} z_{\ell j}\right)\left(x_{m \ell}{ }^{2}+z_{m \ell}{ }^{2}\right)-\left(x_{\ell j}{ }^{2}+y_{\ell j}{ }^{2}\right)\left(y_{m \ell} z_{m \ell}\right)+\left(x_{\ell j} z_{\ell j}\right)\left(x_{m \ell} y_{m \ell}\right)\right.}{\left(r_{l j}{ }^{2} r_{m l}{ }^{2}\right)}, \\
& B=\frac{\left[-\left(x_{\ell j} y_{\ell j}\right)\left(x_{m \ell}{ }^{2}+z_{m \ell}{ }^{2}\right)+\left(x_{\ell j} z_{\ell j}\right)\left(y_{m \ell} z_{m \ell}\right)-\left(y_{\ell j}{ }^{2}+z_{\ell j}{ }^{2}\right)\left(x_{m \ell} y_{m \ell}\right)\right]}{\left(r_{\ell j}{ }^{2} r_{m \ell}{ }^{2}\right)} .
\end{aligned}
$$

In addition, we obtain

$$
W_{H V}^{(3)}=\frac{\left[-\left(x_{\ell j}{ }^{2}+z_{\ell j}{ }^{2}\right)\left(x_{m \ell} y_{m \ell}\right)+\left(y_{\ell j} z_{\ell j}\right)\left(x_{m \ell} z_{m \ell}\right)-\left(x_{\ell j} y_{\ell j}\right)\left(y_{m \ell}{ }^{2}+z_{m \ell}{ }^{2}\right)\right]}{\left(r_{\ell j}{ }^{2} r_{m \ell}{ }^{2}\right)},
$$

and $W_{H H}^{(3)}$ is given by Eq. (A6) with

$$
\begin{gathered}
C=\frac{\left[\left(y_{\ell j} z_{\ell j}\right)\left(x_{m \ell} y_{m \ell}\right)-\left(x_{\ell j}{ }^{2}+y_{\ell j}{ }^{2}\right)\left(x_{m \ell} z_{m \ell}\right)-\left(x_{\ell j} z_{\ell j}\right)\left(y_{m \ell}{ }^{2}+z_{m \ell}{ }^{2}\right)\right]}{\left(r_{\ell j}{ }^{2} r_{m \ell}{ }^{2}\right)}, \\
D=\frac{\left[\left(x_{\ell j} y_{\ell j}\right)\left(x_{m \ell} y_{m \ell}\right)+\left(x_{\ell j} z_{\ell j}\right)\left(x_{m \ell} z_{\ell j}\right)+\left(y_{\ell j}{ }^{2}+z_{\ell j}{ }^{2}\right)\left(y_{m \ell}{ }^{2}+z_{m \ell}{ }^{2}\right)\right]}{\left(r_{\ell j}{ }^{2} r_{m \ell}{ }^{2}\right)} .
\end{gathered}
$$

tal data shown in Figs. 6(b)-6(d) illustrate that the regime of beginning multiple scattering has the potential to be as well understood as are the singlescattering and the deep multiple-scattering regimes.

\section{Appendix A}

For double scattering, using the elements of the matrix $\left[T_{\ell j}\right]$ of Eq. (18) in Eqs. (15), (21), and (23), we obtain

$$
\begin{aligned}
W_{V V}^{(2)} & =\left(x_{\ell j}{ }^{2}+z_{\ell j}{ }^{2}\right) / r_{\ell j}{ }^{2}, \\
W_{V H}^{(2)} & =B \cos (\theta)-A \sin (\theta),
\end{aligned}
$$

with

$$
\begin{gathered}
A=-y_{\ell j} z_{\ell j} / r_{\ell j}{ }^{2}, \\
B=-x_{\ell j} y_{\ell j} / r_{\ell j}{ }^{2} .
\end{gathered}
$$

The generalization of $W_{\alpha \beta}^{(p)}$ to higher orders of scattering is straightforward and requires the multiplication of increasing numbers of $T$ matrices.

The ensemble average of Eq. (29) proceeds as follows: The incoherent portion of Eq. (29) for $p$-order scattering is

$$
\begin{gathered}
\left|\mathbf{E}_{\alpha \beta} *(t+\tau, \theta) \times \mathbf{B}_{\alpha \beta}(t, \theta) / 2 \mu_{0} c\right|^{(p)}=\left(E_{0}{ }^{2} / 2 \mu_{0} c\right) \\
\times\left(1 / r^{2}\right)\left(1 / k^{2 p}\right)\left[\left(n^{2}-1\right)(k a)^{3} /\left(n^{2}+2\right)\right]^{2 p} \\
\quad \times \sum_{\left\{C_{p\}}\right.} \exp \left[i\left(\mathbf{k}_{\mathrm{inc}}-\mathbf{k}_{n m}\right) \cdot \Delta_{n}\right] \ldots \\
\quad \times \exp \left[i\left(\mathbf{k}_{\ell j}-\mathbf{k}_{\mathrm{scatt}}\right) \cdot \Delta_{j}\right] \\
\times\left(W_{\alpha \beta}^{(p)}\right)^{2} /\left(r_{n m}{ }^{2} \ldots r_{\ell j}{ }^{2}\right) .
\end{gathered}
$$


The ensemble average of Eq. (A15) is

$$
\begin{aligned}
\left\langle g_{\alpha \beta}(\tau, \theta)\right\rangle^{(p)}= & \int \mathrm{d}^{3} r_{j} \ldots \int \mathrm{d}^{3} r_{n}(J / V)^{p} \int \mathrm{d}^{3} \Delta_{j} \ldots \\
& \times \int \mathrm{d}^{3} \Delta_{n} P\left(\Delta_{j}\right) \ldots P\left(\Delta_{n}\right) \\
& \times\left|\mathbf{E}_{\alpha \beta} *(t+\tau, \theta) \times \mathbf{B}_{\alpha \beta}(t, \theta) / 2 \mu_{0} c\right|^{(p)},
\end{aligned}
$$

where $V$ is the volume of the sample cell and $P$ is the probability function of Eq. (28). The integration over $\Delta_{i}$ for purely diffusive motion of the suspended particles gives

$$
\int \mathrm{d}^{3} \Delta_{i} \exp \left(i \mathbf{K}_{i} \cdot \Delta_{i}\right) P\left(\Delta_{i}\right)=\exp \left(-K_{i}^{2} D \tau\right)
$$

for $i=\{j, \ldots, n\}$, and the integration over $\mathbf{r}_{j}$ gives

$$
\int \mathrm{d}^{3} r_{j}=V
$$

The remaining $p-1$ integrations over the particle positions $\mathbf{r}_{\ell}, \ldots, \mathbf{r}_{n}$ can be converted to integrals over the relative positions $\mathbf{r}_{\ell j}, \ldots, \mathbf{r}_{n m}$, giving

$$
\begin{aligned}
\left\langle g_{\alpha \beta}(\tau, \theta)\right\rangle^{(p)}= & \left(E_{0}{ }^{2} / 2 \mu_{0} c\right)\left(1 / r^{2}\right)\left(1 / k^{2 p}\right) \\
& \times V(J / V)^{p}\left[\left(n^{2}-1\right)(k a)^{3} /\left(n^{2}+2\right)\right]^{2 p} \\
& \times \int \mathrm{d}^{3} r_{\ell j} \ldots \mathrm{d}^{3} r_{n m} \\
& \times \exp \left(-i\left|\mathbf{k}_{\mathrm{inc}}-\mathbf{k}_{n m}\right|^{2} D \tau\right) \ldots \\
& \times \exp \left(-i\left|\mathbf{k}_{\ell j}-\mathbf{k}_{\mathrm{scatt}}\right|^{2} D \tau\right) \\
& \times\left[W_{\alpha \beta}^{(p)}\right]^{2} /\left(r_{n m}{ }^{2} \ldots r_{\ell j}{ }^{2} .\right.
\end{aligned}
$$

Because of the isotropic-environment assumption, each relative coordinate integration may be written as

$$
\int \mathrm{d}^{3} r_{u v}=\int_{0}^{R_{\text {ave }}} r_{u v}{ }^{2} \mathrm{~d} r_{u v} \int \mathrm{d} \Omega_{u v},
$$

with $u v=\{n m, \ldots, \ell j\}$. Because $W_{\alpha \beta}^{(p)}$ depends on only the angles $\theta_{\ell j}, \phi_{\ell j}, \ldots, \theta_{n m}, \phi_{n m}$ and not on the particle separations $r_{\ell j}, \ldots, r_{n m}$, the radial integrals become

$$
\int_{0}^{R_{\text {ave }}} r_{u v}^{2} \mathrm{~d} r_{u v} /\left(r_{u v}{ }^{2}\right)=R_{\text {ave }}
$$

and Eq. (A19) becomes

$$
\begin{aligned}
\left\langle g_{\alpha \beta}(\tau, \theta)\right\rangle^{(p)}= & \left(E_{0}{ }^{2} / 2 \mu_{0} c\right)\left(1 / r^{2}\right)\left(1 / k^{2 p}\right) \\
& \times(J / V)^{p}\left[\left(n^{2}-1\right)(k a)^{3} /\left(n^{2}+2\right)\right]^{2 p} \\
& \times V\left(R_{\text {ave }}\right)^{p-1} \int \mathrm{d} \Omega_{\ell j} \ldots \mathrm{d} \Omega_{n m} \\
& \times \exp \left(-\left|\mathbf{k}_{\text {inc }}-\mathbf{k}_{n m}\right|^{2} D \tau\right) \ldots \\
& \times \exp \left(-\left|\mathbf{k}_{\ell j}-\mathbf{k}_{\text {scatt }}\right|^{2} D \tau\right) \\
& \times\left[W_{\alpha \beta}^{(p)}\left(\theta, \Omega_{n m}, \ldots, \Omega_{\ell j}\right)\right]^{2} .
\end{aligned}
$$

Using the single-scattering intensity in the $V V$ channel

$$
I_{V V}^{\text {each }}=\left(E_{0}{ }^{2} / 2 \mu_{0} c\right)\left(1 / r^{2}\right)\left[\left(n^{2}-1\right)(k a)^{3} /\left(n^{2}+2\right)\right]^{2}
$$

and the definition of the volume fraction of the suspended particles

$$
f=\left(4 \pi a^{3} / 3\right)(J / V)
$$

causes Eq. (A22) to reduce to Eqs. (30), (33), and (34).

This study was supported in part by the NASAGlenn Research Center under grant NCC3-521. The author wishes to thank William V. Meyer for suggesting this line of inquiry and William V. Meyer and Anthony E. Smart for providing the experimental autocorrelation data. This study is dedicated to the memory of Leslie L. Foldy (26 October 1919-18 January 2001) whose 1945 paper (Ref. 17) created the field of multiple scattering of waves.

\section{References}

1. N. A. Clark, J. H. Lunacek, and G. B. Benedek, "A study of Brownian motion using light scattering," Am. J. Phys. 38, 575-585 (1970).

2. D. A. Weitz and D. J. Pine, "Diffusing-wave spectroscopy," in Dynamic Light Scattering, the Method and Some Applications, W. Brown, ed. (Clarendon, Oxford, UK, 1993), pp. 652-720.

3. H. Schnablegger and O. Glatter, "Sizing of colloidal particles with light scattering: corrections for beginning multiple scattering," Appl. Opt. 34, 3489-3501 (1995).

4. P. Štěpánek, "Static and dynamic properties of multiple light scattering," J. Chem. Phys. 99, 6384-6393 (1993).

5. C. M. Sorensen, R. C. Mockler, and W. J. O'Sullivan, "Depolarized correlation function of light double scattered from a system of Brownian particles," Phys. Rev. A 14, 1520-1532 (1976).

6. C. M. Sorensen, R. C. Mockler, and W. J. O'Sullivan, "Multiple scattering from a system of Brownian particles," Phys. Rev. A 17, 2030-2035 (1978).

7. A. Bøe and O. Lohne, "Dynamical properties of multiply scattered light from independent Brownian particles," Phys. Rev. A 17, 2023-2029 (1978).

8. J. K. G. Dhont, "Multiple Rayleigh-Gans-Debye scattering in colloidal systems: dynamic light scattering," Physica A 129, 374-394 (1985).

9. J. G. Shanks and J. V. Sengers, "Double scattering in critically opalescent fluids," Phys. Rev. A 38, 885-896 (1988).

10. J. A. Lock, "Role of multiple scattering in cross-correlated light scattering with a single laser beam," Appl. Opt. 36, 7559-7570 (1997).

11. F. C. MacKintosh and S. John, "Diffusing wave spectroscopy 
and multiple scattering of light in correlated random media," Phys. Rev. B 40, 2383-2406 (1989).

12. P.-A. Lemieux, M. U. Vera, and D. J. Durian, "Diffusing light spectroscopies beyond the diffusion limit: the role of ballistic transport and anisotropic scattering," Phys. Rev. E 57, 44984515 (1998).

13. A. E. Bailey and D. S. Cannell, "Practical method for calculation of multiple light scattering," Phys. Rev. E 50, 4853-4864 (1994).

14. V. I. Ovod, "Modeling of multiple-scattering suppression by a one-beam cross-correlation system," Appl. Opt. 37, 7856-7864 (1998).

15. Y.-L. Xu, "Electromagnetic scattering by an aggregate of spheres," Appl. Opt. 34, 4573-4588 (1995).

16. Y.-L. Xu, "Electromagnetic scattering by an aggregate of spheres: errata," Appl. Opt. 37, 6494 (1998).

17. L. L. Foldy, "The multiple scattering of waves," Phys. Rev. 67, 107-119 (1945).

18. A. Ishimaru, Wave Propagation and Scattering in Random Media (Academic, New York, 1978), Vol. 2, pp. 254-256.

19. S. Stein, "Addition theorems for spherical wave functions," $Q$. Appl. Math. 19, 15-24 (1961).

20. O. R. Cruzan, "Translation addition theorems for spherical vector wave functions," Q. Appl. Math. 20, 33-40 (1962).

21. Y.-L. $\mathrm{Xu}$, "Electromagnetic scattering by an aggregate of spheres: far zone," Appl. Opt. 36, 9496-9508 (1997).

22. H. C. van de Hulst, Light Scattering by Small Particles (Dover, New York, 1981), p. 144.
23. D. J. Griffiths, Introduction to Electrodynamics, 3rd ed. (Prentice-Hall, Upper Saddle River, N.J., 1999), p. 158.

24. E. M. Purcell and C. R. Pennypacker, "Scattering and absorption of light by nonspherical dielectric grains," Astrophys. J. 186, 705-714 (1973).

25. S. B. Singham and C. F. Bohren, "Light scattering by an arbitrary particle: a physical reformulation of the coupled dipole method," Opt. Lett. 12, 10-12 (1987).

26. S. B. Singham and C. F. Bohren, "Light scattering by an arbitrary particle: the scattering-order formulation of the coupled-dipole method,” J. Opt. Soc. Am. A 5, 1867-1872 (1988).

27. A. R. Jones, "Electromagnetic wave scattering by assemblies of particles in the Rayleigh approximation," Proc. R. Soc. London Ser. A 366, 111-127 (1979).

28. W. Lou and T. T. Charalampopoulos, "On the electromagnetic scattering and absorption of agglomerated small spherical particles," J. Phys. D 27, 2258-2270 (1994).

29. V. Twersky, "On scattering of waves by random distributions. I. Free-space scatterer formalism," J. Math. Phys. 3, 700715 (1962).

30. W. V. Meyer, D. S. Cannell, A. E. Smart, T. W. Taylor, and P. Tin, "Multiple-scattering suppression by cross correlation," Appl. Opt. 36, 7551-7558 (1997).

31. F. C. MacKintosh, J. X. Zhu, D. J. Pine, and D. A. Weitz, "Polarization memory of multiply scattered light," Phys. Rev. B 40, 9342-9345 (1989). 